\title{
Experimental Study on the Influence on Vibration Characteristics of Thin Cylindrical Shell with Hard Coating under Cantilever Boundary Condition
}

\author{
Hui Li, Wei Sun, Mingwei Zhu, and Pengcheng Xue \\ School of Mechanical Engineering and Automation, Northeastern University, No. 3-11 Wenhua Road, Heping District, \\ Shenyang 110819, China
}

Correspondence should be addressed to Hui Li; lh200300206@163.com

Received 12 February 2017; Accepted 15 May 2017; Published 3 July 2017

Academic Editor: Athanasios Chasalevris

Copyright (C) 2017 Hui Li et al. This is an open access article distributed under the Creative Commons Attribution License, which permits unrestricted use, distribution, and reproduction in any medium, provided the original work is properly cited.

\begin{abstract}
This research has experimentally investigated the influence on vibration characteristics of thin cantilever cylindrical shell (TCS) with hard coating under cantilever boundary condition. Firstly, the theoretical model of TCS with hard coating is established to calculate its natural frequencies and modal shapes so as to roughly understand vibration characteristic of TCS when it is coated with hard coating material. Then, by considering its nonlinear stiffness and damping influences, an experiment system is established to accurately measure vibration parameters of the shell, and the corresponding test methods and identification techniques are also proposed. Finally, based on the measured data, the influences on natural frequencies, modal shapes, damping ratios, and vibration responses of TCS with hard coating are analyzed and discussed in detail. It can be found that hard coating can play an important role in vibration reduction of TCS, and for the most modes of TCS, hard coating will result in the decrease of natural frequencies, but the decreased level is not very big, and its damping effects on the higher frequency range of the shell are weak and ineffective. Therefore, in order to make better use of this coating material, we must carefully choose the concerned antivibration frequency range of the shell; otherwise it may lead to some negative effects.
\end{abstract}

\section{Introduction}

Thin cylindrical shell (TCS) has long been an important structural component due to its high stiffness to weight and strength to weight ratios, which is widely used in the engineering fields, such as aircraft casings, pipes and ducts, rotary drums in granulator, and aircraft engine $[1,2]$. TCS is often working in complex-field coupling environment, such as vibration, noise, high temperature, and high-speed flow erosion, which may easily cause high level vibration and fatigue failure and lead to severe security accidents [3-5]. Therefore, it is of great scientific significance to study how to reduce and suppress the vibration of TCS.

The hard coating is a kind of coating materials prepared by the metal, ceramic, or their mixtures, which is used as surface treatment for antifriction, antierosion, vibration reduction, and other engineering application fields [6-8]. In recent years, due to continuous reduced costs and more mature technologies, it has drawn increasingly academic and engineering attention. Usually this damping material is coated on the surface of TCS; through the internal friction of coated particle and interface friction of the coating and metallic substrate, the structural vibration energy is finally transformed into heat energy which will be dissipated in the composite structural system $[9,10]$, so that the effect of restraining and reducing vibration of TCS can be realized.

At present, great efforts have been made to study vibration character of thin-walled structures with hard coating, such as beams, plates, and shells including the corresponding modeling and analysis techniques, antivibration design, and evaluation methods. For example, Ivancic et al. [11, 12] measured inherent characteristic of titanium plate coated with magnesium aluminate spinel; they found, compared with the uncoated specimen, frequency response of hard coating composite plate was not symmetric about the resonance frequency, and the resonance frequency decreased as the 
amplitude of the applied force was increased, indicating some characteristics of nonlinear stiffness, and they called this phenomenon "strain softening." Green and Patsias [13] proposed a friction model to calculate the response of a coated beam and the variation of damping effectiveness for several vibration modes, and a range of amplitudes was calculated, which showed this model can capture the behaviors of the test piece well after comparison with experimental results. Jang et al. [14] analyzed the stress distribution of cylindrical shell structure with thermal barrier coating by finite element method. The results showed that the radial stress of the coating decreased with curvature, and the circumferential stress increased at first and then decreased during cooling time. Song et al. [15] studied high order vibration characteristics of rotating thin shells and hard coating damping effects. Transfer matrix method is used to calculate the high order modes and the moving wave characteristics of the shell, and it was observed that high order vibration characteristics of rotating thin shells and hard coating damping material had a more obvious effect on the natural frequency at the high vibration order. Najafov et al. [16] investigated linear and nonlinear vibrations of a truncated conical shell covered with functionally graded coatings, and parametric studies are performed to illustrate the effect of different values of thickness and coating material on the frequency-amplitude relations. Sofiyev [17] investigated the vibration and buckling of cylindrical shells covered by different coatings, such as functionally graded, metal, and ceramic coatings. The equations of motion of such composite shells are deduced by using first-order shear deformation theory, and the influences of the compositional profiles of coatings, the shear stresses, the radius-to-thickness ratio, and so on are discussed in detail.

Besides, many scholars also studied nonlinear vibration analysis methods for composite cylindrical shells, and these analytical techniques can provide important reference for studying vibration characteristics of the shell with coating materials. For example, Li et al. [18] studied the nonlinear vibration of laminated cylindrical shell with initial imperfections by combining higher-order shear deformation theory with Kármán-Donnell-type of kinematic nonlinearity. Singh and Panda [19] investigated the nonlinear vibration behavior of laminated composite shell under uniform temperature load. Ribeiro [20] investigated the nonlinear vibration of thin laminated shell with curvilinear fibers by higher-order shear deformation theory and the $p$-version finite element method. Mahapatra et al. [21] investigated nonlinear vibration of laminated composite shell under hygrothermal environment by higher-order shear deformation theory and discretized finite element method. Kar and Panda [22] studied geometrical nonlinear vibration behavior of functionally graded spherical shell by using Green-Lagrange nonlinear kinematics in the framework of the higher-order shear deformation theory. Mahapatra and Panda [23] investigated the nonlinear vibration of laminated composite spherical shell by using the midplane kinematics of higher-order shear deformation theory and Green-Lagrange type geometric nonlinearity.

However, most of researches done by the above scholars and researchers are mainly based on theory or simulation, experimental studies on the influence on vibration characteristics of TCS with hard coating are still scarce, and as a lack of the related test conclusion, theoretical analysis result cannot be effectively verified, let alone validating some advanced composite shell theories. Besides, due to the complicated vibrational properties of TCS, such as very closed modes, the small vibration levels and abundant local vibration, especially when the hard coating material is applied on its surface, such composite shell structure is turned into a nonlinear system with variable stiffness and damping under different excitation levels, which would inevitably increase the experimental difficulties. Up until now, either from the perspective of test accuracy, or from the test efficiency, these test problems have not been well solved. In order to meet the needs of engineering applications of such damping material, it is necessary to adopt some experimental techniques to study vibration characteristics of TCS with hard coating.

In this research, the influence on vibration characteristics of thin cylindrical shell with hard coating has been investigated experimentally under cantilever boundary condition. Firstly in Section 2, the theoretical model of TCS with hard coating is established to calculate its natural frequencies and modal shapes to roughly understand vibration characteristic of TCS when it is coated with hard coating material. Then in Section 3 we go on to set up the experiment system to accurately measure vibration parameters of TCS with hard coating. By considering its nonlinear stiffness and damping influences, the experimental test is divided into two phases, that is, Phase I: no coating TCS measurement and Phase II: coated TCS measurement, and the corresponding test methods and identification techniques are also proposed. Next in Section 4, by using the proposed method, such as the smallsegment FFT processing technique, the sliding-envelope method and laser rotating scanning method, natural frequencies, modal shapes, damping ratios, and vibration responses of TCS under coating and no coating states are well measured. Finally in Section 5, based on the accurate measured data, the influence of hard coating on vibration characteristics of cantilever cylindrical shell is analyzed and discussed in detail, and we also compare the calculated frequency results with the measured ones. This research can provide dynamic modeling service for TCS with hard coating, provide experimental test methods to obtain nonlinear vibration parameters with high efficiency and accuracy, and also provide an important reference for analysis and diagnosis of vibration fault of composite TCS.

\section{Vibration Characteristic Analysis of TCS with Hard Coating}

In this section, in order to deeply understand vibration characteristic of TCS with hard coating, the natural frequencies and modal shapes of coated TCS are calculate based on the established theoretical model. Although this model can not calculate damping results and also may inevitably contain some calculation errors, it is helpful for us to determine measured frequency range, build experimental model, understand geographic distributions of some nodes or nodal lines, and so forth. 


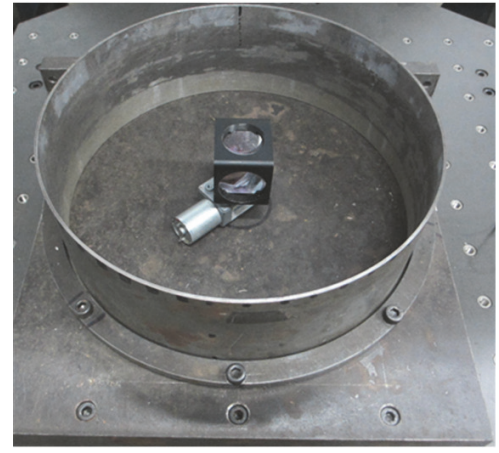

Thin cylindrical shell
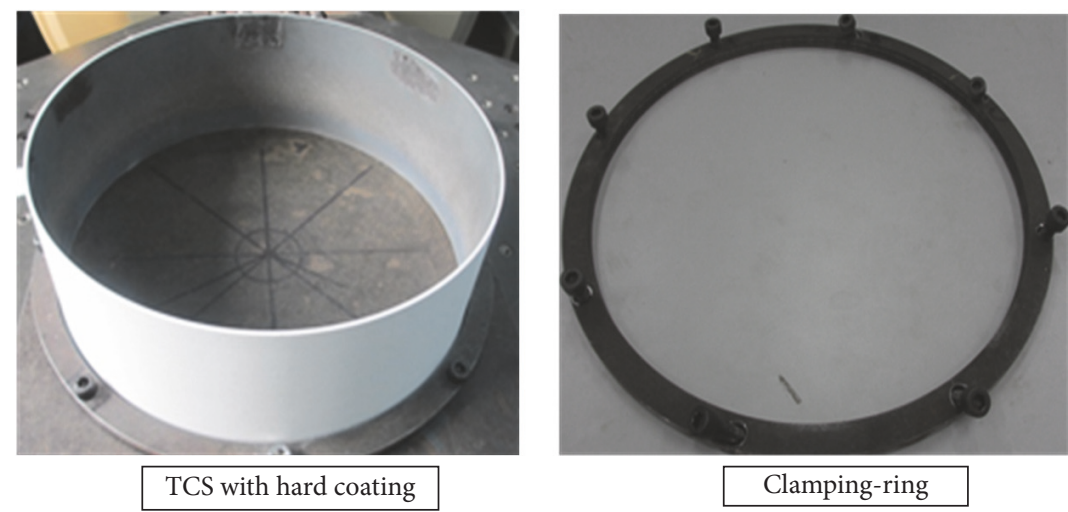

FIGURE 1: TCS coated with and without hard coating and its clamping-ring used in cantilever boundary condition.

TABLE 1: Dimension parameters of thin cylindrical shell.

\begin{tabular}{lc}
\hline Dimension name & Dimension values \\
\hline Length $(\mathrm{mm})$ & 100 \\
Thickness $(\mathrm{mm})$ & 2 \\
Internal radius $(\mathrm{mm})$ & 142 \\
External radius $(\mathrm{mm})$ & 144 \\
Extension edge radius $(\mathrm{mm})$ & 150 \\
Thickness of extension edge $(\mathrm{mm})$ & 3 \\
\hline
\end{tabular}

TABLE 2: Material parameters of TCS and hard coating.

\begin{tabular}{lccc}
\hline Name & $\begin{array}{c}\text { Elastic modulus } \\
(\mathrm{Pa})\end{array}$ & Poisson's ratio & $\begin{array}{c}\text { Density } \\
\left(\mathrm{kg} / \mathrm{m}^{3}\right)\end{array}$ \\
\hline Structural steel & $2.12 \times 10^{11}$ & 0.3 & 7850 \\
NiCrAlCoY + YSZ & $5.45 \times 10^{10}$ & 0.3 & 4176 \\
\hline
\end{tabular}

2.1. Research Object. The TCS studied in this research is made of structural steel with the length of $100 \mathrm{~mm}$ and an average thickness of $2 \mathrm{~mm}$, as shown in Figure 1; its specific dimension parameters and material parameters are listed in Tables 1 and 2, respectively. There is the extension edge with $150 \mathrm{~mm}$ external radius and $3 \mathrm{~mm}$ thickness on this shell which is machined to be clamped by a clamping-ring with eight M8 bolts, so that it can be ensured that the shell is under cantilever boundary condition. Then, we can coat hard coating material onto the outside surface of the shell by plasma spraying technique; the coating is composed of NiCrAlCoY + YSZ with the thickness of about $0.31 \mathrm{~mm}$, and the material parameters are also listed in Table 2.

2.2. Vibration Characteristic Analysis of TCS with Hard Coating. The theoretical model of TCS with hard coating is shown in Figure 2, its axial length is $L$, and $u, v, w$ are the displacement of any point on the middle surface in the $x, \theta, z$ direction, respectively. Then, the curvilinear coordinates system $o-x \theta z$ is used in this model, which is located

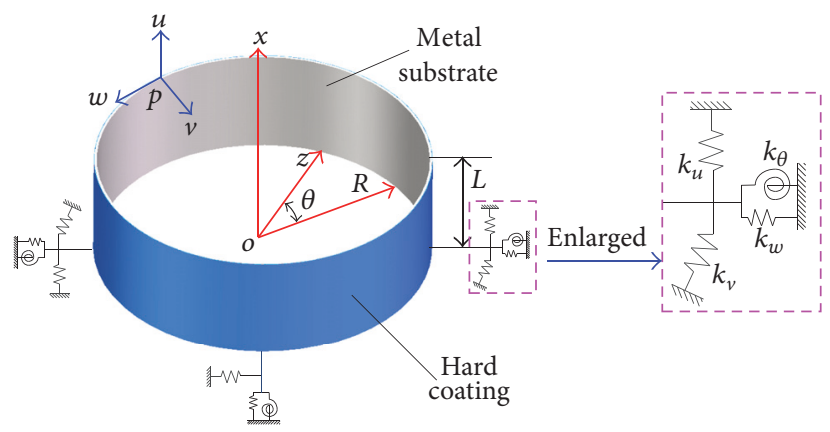

FIGURE 2: The theoretical model of TCS with hard coating.

in the center point of restrained end, and some distribution of springs in the clamped end is employed to simulate actual fixed boundary of TCS, whose support stiffness is represented by $k_{u}, k_{v}$, and $k_{w}$ in $u, v$, and $w$ direction while $k_{\theta}$ is the rotation support stiffness. Firstly, according to Kirchhoff-Love assumption, the transverse shear deformation and transverse extrusion deformation can be ignored, and its transverse extrusion stress can also be ignored at the same time. Then, TCS can be regarded as many thin layers which are parallel to the mid-surface, and these thin layers can deform independently yet keep the straight normal characteristic. Thus, the vibration characteristic of whole shell can be studied through the mid-surface deformation and vibration.

The cross section of TCS with hard coating is shown in Figure 3, and the radius of middle surface is $R$ while the thickness of substrate and hard coating is $T_{s}$ and $T_{c}$, respectively. Besides, $h_{0}$ represents the distance of middle surface and inside wall of coated TCS, which can be expressed as

$$
h_{0}=\frac{E_{s} T_{s}^{2}+2 E_{c} T_{s} T_{c}+E_{c} T_{c}^{2}}{2\left(E_{s} T_{s}+E_{c} T_{c}\right)},
$$




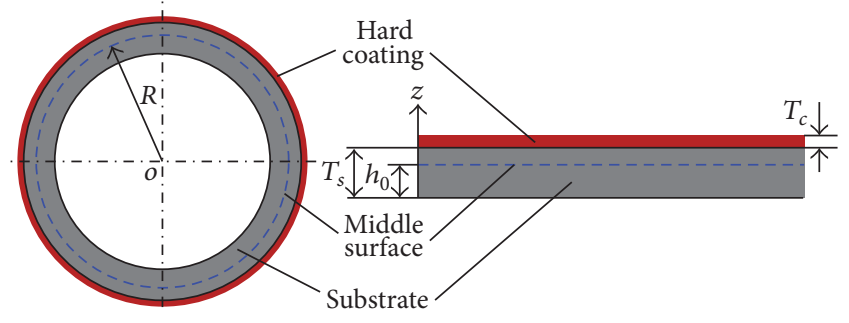

FIGURE 3: Schematic of cross section of TCS with hard coating.

The strain-displacement relations of TCS with hard coating can be expressed as

$$
\begin{gathered}
\varepsilon_{x}=\varepsilon_{1}+z k_{1}, \\
\varepsilon_{\theta}=\varepsilon_{2}+z k_{2}, \\
\gamma_{x \theta}=\gamma+2 z \tau,
\end{gathered}
$$

where

$$
\begin{aligned}
\varepsilon_{1} & =\frac{\partial u}{\partial x} \\
\varepsilon_{2} & =\frac{1}{R}\left(\frac{\partial v}{\partial \theta}+w\right) \\
\gamma & =\frac{\partial v}{\partial x}+\frac{1}{R} \frac{\partial u}{\partial \theta} \\
k_{1} & =\frac{\partial^{2} w}{\partial x^{2}} \\
k_{2} & =-\frac{1}{R^{2}}\left(\frac{\partial^{2} w}{\partial \theta^{2}}-\frac{\partial v}{\partial \theta}\right), \\
\tau & =-\frac{1}{R}\left(2 \frac{\partial^{2} w}{\partial x \partial \theta}-\frac{\partial v}{\partial x}\right),
\end{aligned}
$$

where $\varepsilon_{1}, \varepsilon_{2}$, and $\gamma$ represent the axial strain, tangential strain, and shear strain of any point on the middle surface, respectively. Meanwhile, $k_{1}, k_{2}$, and $\tau$ are the curvature of middle surface of the shell.

Assuming that the TCS is under the plane stress state, then on the basis of generalized Hooke law its strain-stress relations can be described as

$$
\boldsymbol{\sigma}=\mathbf{Q} \boldsymbol{\varepsilon}
$$

where

$$
\begin{aligned}
\boldsymbol{\sigma}^{\mathrm{T}} & =\left\{\begin{array}{lll}
\sigma_{x} & \sigma_{\theta} & \sigma_{x \theta}
\end{array}\right\} \\
\mathbf{Q} & =\left[\begin{array}{ccc}
Q_{11} & Q_{12} & 0 \\
Q_{12} & Q_{22} & 0 \\
0 & 0 & Q_{66}
\end{array}\right] \\
\boldsymbol{\varepsilon}^{\mathrm{T}} & =\left\{\begin{array}{lll}
\varepsilon_{x} & \varepsilon_{\theta} & \gamma_{x \theta}
\end{array}\right\},
\end{aligned}
$$

where $\boldsymbol{\sigma}$ and $\boldsymbol{\varepsilon}$ represent stress and strain vector; $\mathbf{Q}$ is the elastic matrix.
The hard coating and substrate are all isotropic materials, so the elastic matrix of them can be defined as

$$
\begin{aligned}
Q_{11} & =\frac{E}{1-\mu^{2}}, \\
Q_{12} & =\frac{E v}{1-\mu^{2}}, \\
Q_{22} & =\frac{E}{1-\mu^{2}}, \\
Q_{66} & =\frac{E}{2(1+\mu)},
\end{aligned}
$$

where $E$ is elastic modulus; $\mu$ is Poisson's ratio. For the hard coating, we know that $E=E_{c}, \mu=\mu_{c}$, and for the substrate, $E=E_{s}, \mu=\mu_{s}$ should be used.

The stress resultants and bending moment resultants of TCS with hard coating can be expressed as

$$
\begin{gathered}
\left(N_{x}, N_{\theta}, N_{x \theta}\right)=\int_{-h_{0}}^{T_{s}+T_{c}-h_{0}}\left(\sigma_{x}, \sigma_{\theta}, \sigma_{x \theta}\right) d z \\
\left(M_{x}, M_{\theta}, M_{x \theta}\right)=\int_{-h_{0}}^{T_{s}+T_{c}-h_{0}}\left(\sigma_{x}, \sigma_{\theta}, \sigma_{x \theta}\right) z d z,
\end{gathered}
$$

where $N_{x}, N_{\theta}$, and $N_{x \theta}$ represent the stress resultants of TCS in the $x, \theta, z$ direction, respectively, while $M_{x}, M_{\theta}$, and $M_{x \theta}$ are the related bending moment resultants.

Substituting (4) into (7), the constitutive equation can be obtained:

$$
\mathbf{N}=\mathbf{S} \boldsymbol{\xi}
$$

where

$$
\begin{aligned}
\mathbf{N}^{\mathrm{T}} & =\left\{\begin{array}{llllll}
N_{x} & N_{\theta} & N_{x \theta} & M_{x} & M_{\theta} & M_{x \theta}
\end{array}\right\} \\
\boldsymbol{\xi}^{\mathrm{T}} & =\left\{\begin{array}{lllllll}
\varepsilon_{1} & \varepsilon_{2} & \gamma & k_{1} & k_{2} & 2 \tau
\end{array}\right\} \\
\mathbf{S} & =\left[\begin{array}{cccccc}
A_{11} & A_{12} & 0 & B_{11} & B_{12} & 0 \\
A_{12} & A_{22} & 0 & B_{12} & B_{22} & 0 \\
0 & 0 & A_{66} & 0 & 0 & B_{66} \\
B_{11} & B_{12} & 0 & D_{11} & D_{12} & 0 \\
B_{12} & B_{22} & 0 & D_{12} & D_{22} & 0 \\
0 & 0 & B_{66} & 0 & 0 & D_{66}
\end{array}\right],
\end{aligned}
$$

where $A_{i j}$ is tension stiffness, $B_{i j}$ is coupling stiffness, and $D_{i j}$ is bending stiffness, $i, j=1,2,6$, which have the following form:

$$
\begin{aligned}
& A_{i j}=\int_{-h_{0}}^{T_{s}-h_{0}} Q_{i j}^{s} d z+\int_{T_{s}-h_{0}}^{T_{s}+T_{c}-h_{0}} Q_{i j}^{c} d z \\
& B_{i j}=\int_{-h_{0}}^{T_{s}-h_{0}} Q_{i j}^{s} z d z+\int_{T_{s}-h_{0}}^{T_{s}+T_{c}-h_{0}} Q_{i j}^{c} z d z \\
& D_{i j}=\int_{-h_{0}}^{T_{s}-h_{0}} Q_{i j}^{s} z^{2} d z+\int_{T_{s}-h_{0}}^{T_{s}+T_{c}-h_{0}} Q_{i j}^{c} z^{2} d z,
\end{aligned}
$$


where $Q_{i j}^{s}$ and $Q_{i j}^{c}$ are the elastic matrix elements of substrate and hard coating, respectively. After integrating, (10) can be simplified as

$$
\begin{aligned}
A_{i j} & =Q_{i j}^{s}\left(h_{1}-h_{0}\right)+Q_{i j}^{c}\left(h_{2}-h_{1}\right) \\
B_{i j} & =\frac{1}{2} Q_{i j}^{s}\left(h_{1}^{2}-h_{0}^{2}\right)+Q_{i j}^{c}\left(h_{2}^{2}-h_{1}^{2}\right) \\
D_{i j} & =\frac{1}{3} Q_{i j}^{s}\left(h_{1}^{3}-h_{0}^{3}\right)+Q_{i j}^{c}\left(h_{2}^{3}-h_{1}^{3}\right),
\end{aligned}
$$

where $h_{1}$ represents the distance between the middle surface and the bonding surface of substrate and hard coating, while $h_{2}$ is the distance of middle surface and outside wall of coated TCS.

Based on the theoretical model above, the strain energy $U$, kinetic energy $T$, and the elastic potential energy of the spring $U_{\text {sp }}$ can be expressed as

$$
\begin{aligned}
U & =\frac{1}{2} \int_{0}^{L} \int_{0}^{2 \pi} \xi^{\mathrm{T}} S \boldsymbol{\xi} R d \theta d x \\
T & =\frac{1}{2} \\
& \cdot \int_{0}^{L} \int_{0}^{2 \pi} \rho_{\mathrm{T}} R\left[\left(\frac{\partial u}{\partial t}\right)^{2}+\left(\frac{\partial v}{\partial t}\right)^{2}+\left(\frac{\partial w}{\partial t}\right)^{2}\right] d \theta d x \\
U_{\mathrm{sp}} & =\frac{1}{2} \\
& \cdot \int_{0}^{2 \pi}\left\{k_{u} u^{2}+k_{v} v^{2}+k_{w} w^{2}+k_{\theta}\left(\frac{\partial w}{\partial x}\right)^{2}\right\} R d \theta,
\end{aligned}
$$

where

$$
\rho_{\mathrm{T}}=\int_{-h_{0}}^{T_{s}-h_{0}} \rho_{s} d z+\int_{T_{s}-h_{0}}^{T_{s}+T_{c}-h_{0}} \rho_{c} d z
$$

where $\rho_{s}$ and $\rho_{c}$ are the density of the substrate and hard coating, respectively.

Assume that, in the $x, \theta, z$ direction, the displacement of any point $p$ of coated TCS on the middle surface can be expressed as

$$
\begin{aligned}
u & =U(\kappa) \cos (n \theta) \sin (\omega t) \\
v & =V(\kappa) \sin (n \theta) \sin (\omega t) \\
w & =W(\kappa) \cos (n \theta) \sin (\omega t),
\end{aligned}
$$

where $n$ represents the circumferential waves in $\theta$ direction of coated TCS, $\omega$ is natural frequency and $U(\kappa), V(\kappa)$, and $W(\kappa)$ are modal shape functions in $x, \theta, z$ direction of coated TCS, which can be expressed as

$$
\begin{aligned}
U(\kappa) & =\sum_{m=1}^{N T} a_{m} P_{m}^{u}(\kappa) \\
V(\kappa) & =\sum_{m=1}^{N T} b_{m} P_{m}^{v}(\kappa) \\
W(\kappa) & =\sum_{m=1}^{N T} c_{m} P_{m}^{w}(\kappa),
\end{aligned}
$$

where, $a_{m}, b_{m}$, and $c_{m}$ are the shape coefficients; NT represents the sum number in actual calculation. Besides, $P^{u}(\kappa), P^{v}(\kappa)$, and $P^{w}(\kappa)$ are the displacement admissible functions of any point on the middle surface in the $x, \theta, z$ direction, and $\kappa=$ $x / L$.

This is because the displacement admissible function can be obtained from the Gram-Schmidt process. When the first polynomial $p_{1}^{\Delta}(\kappa)(\Delta=u, v, w)$ are settled, a series of orthogonal polynomial functions can be obtained:

$$
\begin{array}{rl}
p_{2}^{\Delta}(\kappa) & =\left(\kappa-B_{2}\right) p_{1}^{\Delta}(\kappa) \\
p_{k}^{\Delta}(\kappa) & =\left(\kappa-B_{k}\right) p_{k-1}^{\Delta}(\kappa)-C_{k} p_{k-2}^{\Delta}(\kappa) \\
k & k=1,2, \ldots, N T,
\end{array}
$$

where

$$
\begin{aligned}
B_{k} & =\frac{\int_{a}^{b} \kappa \psi(\kappa)\left[p_{k-1}^{\Delta}(\kappa)\right]^{2} d \kappa}{\int_{a}^{b} \psi(\kappa)\left[p_{k-1}^{\Delta}(\kappa)\right]^{2} d \kappa} \\
C_{k} & =\frac{\int_{a}^{b} \kappa \psi(\kappa) p_{k-1}^{\Delta}(\kappa) p_{k-2}^{\Delta}(\kappa) d \kappa}{\int_{a}^{b} \psi(\kappa)\left[p_{k-2}^{\Delta}(\kappa)\right]^{2} d \kappa},
\end{aligned}
$$

where $\psi(\kappa)$ is the weighting function.

In order to finish the simulation work of cantilever boundary condition of TCS, as seen in Figure 2, firstly, we simulate the free-free boundary condition

$$
p_{1}^{\Delta}(\kappa)=1, \quad(\Delta=u, v, w) .
$$

After that, the orthogonal polynomial functions can be settled. And we chose $k_{u}=k_{v}=k_{w}=k_{\theta}=10^{7}$ to simulate actual constraint boundary condition in Figure 3. Then, substituting displacement equation (16) into strain energy equation (12), kinetic energy equation (13), and elastic potential energy equation (14), we can get

$$
\begin{aligned}
U & =\frac{L \pi}{2 R}[\cos (\omega t)]^{2} \int_{0}^{1}\left\{n^{2} A_{66} U^{2}\right. \\
& +\frac{R^{2} A_{11}}{L^{2}}\left(\frac{\partial U}{\partial \kappa}\right)^{2} d \kappa+\left(\frac{2 n R A_{12}}{L}+\frac{2 n B_{12}}{L}\right) \frac{\partial U}{\partial \kappa} V \\
& -\left(\frac{2 n R A_{66}}{L}+\frac{2 n B_{66}}{L}\right) \frac{\partial V}{\partial \kappa} U+\frac{2 n^{2} B_{12}}{L} \frac{d U}{d \kappa} W \\
& -\frac{4 n^{2} B_{66}}{L} \frac{d W}{d \kappa} U+\frac{2 R A_{12}}{L} \frac{d U}{d \kappa} W \\
& -\frac{2 R^{2} B_{11}}{L^{3}} \frac{d U}{d \kappa} \frac{d^{2} W}{d \kappa^{2}} \\
& +\left(n^{2} A_{22}+\frac{2 n^{2} B_{22}}{R}+\frac{n^{2} D_{22}}{R^{2}}\right) V^{2} \\
& +\left(\frac{4 D_{66}}{L^{2}}+\frac{4 R B_{66}}{L^{2}}+\frac{R^{2} A_{66}}{L^{2}}\right)\left(\frac{d V}{d \kappa}\right)^{2}
\end{aligned}
$$




$$
\begin{aligned}
+ & \left(\frac{4 n R B_{66}}{L^{2}}+\frac{8 n D_{66}}{L^{2}}\right) \frac{d V}{d \kappa} \frac{d W}{d \kappa} \\
& -\left(\frac{2 n R B_{12}}{L^{2}}+\frac{2 n D_{12}}{L^{2}}\right) V \frac{d^{2} W}{d \kappa^{2}} \\
+ & \left(2 n A_{22}+\frac{2 n^{3} B_{22}}{R}+\frac{2 n B_{22}}{R}+\frac{2 n^{3} D_{22}}{R^{2}}\right) V W \\
+ & \left(A_{22}+\frac{2 n^{2} B_{22}}{R}+\frac{n^{4} D_{22}}{R^{2}}\right) W^{2} \\
+ & \frac{4 n^{2} D_{66}}{L^{2}}\left(\frac{d W}{d \kappa}\right)^{2}+\frac{R^{2} D_{11}}{L^{4}}\left(\frac{d^{2} W}{d \kappa^{2}}\right)^{2} \\
T= & \frac{\pi \rho_{t} L R}{2} \omega^{2}[\cos (\omega t)]^{2} \int_{0}^{1}\left(U^{2}+V^{2}+W^{2}\right) d \kappa \\
+ & \left.\left.\frac{2 n_{12}}{L^{2}}+\frac{2 R B_{12}}{L^{2}}\right) W\left(\frac{d^{2} W}{d \kappa^{2}}\right)\right\} d \kappa \\
U_{\text {sp }} & \left.\left.\left.=\frac{\partial W}{2}\right)^{2}\right] \cdot \cos (\omega t)\right]^{2}\left[k_{u} U^{2}+k_{v} V^{2}+k_{w} W^{2}\right. \\
& k_{6}
\end{aligned}
$$

According to Rayleigh-Ritz method, the Lagrange function $\Pi$ can be used to define the difference between the maximum strain energy and the maximum kinetic energy, which can be expressed as

$$
\Pi=\left(U+U_{\mathrm{sp}}\right)_{\max }-T_{\max }
$$

Then, take the partial of shape coefficients $a_{m}, b_{m}$, and $c_{m}$ of Lagrange function $\Pi$ and set the value equal to zero; we can get

$$
\frac{\partial \Pi}{\partial \alpha}=0
$$

where $\alpha=a_{1}, a_{2}, \ldots, a_{N T}, b_{1}, b_{2}, \ldots, b_{N T}, c_{1}, c_{2}, \ldots, c_{N T}$.

Furthermore, the equation which contains the shape coefficients of $a_{m}, b_{m}$, and $c_{m}$ and the corresponding sum number can reach $3 N T$, which can be expressed as

$$
\left(\mathbf{K}+\mathbf{K}_{\mathrm{sp}}-\omega^{2} \mathbf{M}\right) \mathbf{X}=0
$$

where $\mathbf{X}$ is characteristic vector, having the following form:

$$
\mathbf{X}^{\mathrm{T}}=\left\{a_{1}, a_{2}, \ldots, a_{N T}, b_{1}, b_{2}, \ldots, b_{N T}, c_{1}, c_{2}, \ldots, c_{N T}\right\}
$$

Besides, in (24), $\mathbf{K}, \mathbf{K}_{\mathrm{sp}}$, and $\mathbf{M}$ are stiffness matrix, the spring stiffness matrix, and mass matrix of coated TCS, and the dimensions of these matrixes are $3 N T \times 3 N T$, which can be expressed as

$$
\begin{aligned}
\mathbf{K} & =\left[\begin{array}{lll}
\mathbf{K}^{11} & \mathbf{K}^{12} & \mathbf{K}^{13} \\
\mathbf{K}^{21} & \mathbf{K}^{22} & \mathbf{K}^{23} \\
\mathbf{K}^{31} & \mathbf{K}^{32} & \mathbf{K}^{33}
\end{array}\right] \\
\mathbf{K}_{\mathrm{sp}} & =\left[\begin{array}{lll}
\mathbf{K}_{\mathrm{sp}}^{11} & & \\
& \mathbf{K}_{\mathrm{sp}}^{22} & \\
& & \mathbf{K}_{\mathrm{sp}}^{33}
\end{array}\right] \\
\mathbf{M} & =\left[\begin{array}{lll}
\mathbf{M}^{11} & & \\
& \mathbf{M}^{22} & \\
& & \mathbf{M}^{33}
\end{array}\right] .
\end{aligned}
$$

It should be noted that each submatrix in the above matrixes is $N T \times N T$, and it can be seen in the Appendix. Then, by solving characteristic equation (24), the natural frequencies and characteristic vectors can be obtained. At the same time, substituting the characteristic vector $\mathbf{X}$ which is related to any circumferential wave $n$ into displacement equation (16), we can obtain the corresponding mode shape results, which are the combination of the circumferential wave $n$ and the axial half wave $m$.

Finally, repeat the above steps, and the natural frequencies and mode shapes of cantilever cylindrical shell with and without hard coating can be gradually calculated out based on the self-written Matlab program, which are listed in Table 3. It can be found from the calculated results in Table 3 that (I) hard coating will result in the decrease of its natural frequencies, but the decreased level is not very big, which is within the range of $0.4 \% \sim 1.9 \%$; (II) high order natural frequencies of the shell, for example, the 7 th and the 8 th natural frequency, basically will not be affected by the hard coating material and the varied frequencies are less than $0.5 \%$; (III) as the hard coating material is very thin, the mode shapes of the shell cannot be easily changed by such coating. It should be noted that due to the complexity of the damping between the coating and the metal substrate of TCS, we do not introduce damping parameters to the established theoretical model, so its vibration response results are not calculated in this section.

\section{Test System and Method of Vibration Characteristics of TCS with Hard Coating}

In Section 2, the vibration characteristic of hard coating composite shell is roughly analyzed. But because the nonlinear stiffness and damping characteristics are very complex, which cannot be accurately analyzed by simulation method, it is necessary to employ experimental technique to investigate the influence on vibration characteristics of TCS with hard coating. In this section, an experiment system is firstly established to accurately measure vibration parameters of the shell, and the corresponding test procedures and identification 
TABLE 3: The calculated natural frequencies and mode shapes of cantilever cylindrical shell with and without hard coating.

\begin{tabular}{|c|c|c|c|c|c|}
\hline \multirow[b]{2}{*}{ Modal order } & \multicolumn{2}{|c|}{ TCS without coating } & \multicolumn{2}{|c|}{ TCS with hard coating } & \multirow[b]{2}{*}{$\begin{array}{l}\text { Frequency differences } \\
\qquad(B-A) / A(\%)\end{array}$} \\
\hline & $\begin{array}{c}\text { Natural frequency } \\
A(\mathrm{~Hz})\end{array}$ & Mode shape $(m, n)$ & $\begin{array}{c}\text { Natural frequency } \\
B(\mathrm{~Hz})\end{array}$ & Mode shape $(m, n)$ & \\
\hline \multirow[t]{2}{*}{1} & 923.1 & 60 & 918.4 & 01 & 0.5 \\
\hline & & $(1,5)$ & & $(1,5)$ & \\
\hline \multirow[t]{3}{*}{2} & 985.3 & 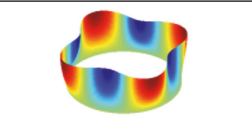 & 972.3 & Oe & 1.3 \\
\hline & & $(1,4)$ & & $(1,4)$ & \\
\hline & 1064.9 & got & 1057.8 & gon & 0.7 \\
\hline 3 & & $(1,6)$ & & $(1,6)$ & \\
\hline \multirow[t]{2}{*}{4} & 1194.6 & & 1171.8 & & 1.9 \\
\hline & & $(1,3)$ & & $(1,3)$ & \\
\hline \multirow[t]{2}{*}{5} & 1326.5 & (201) & 1306.3 & (2001) & 1.5 \\
\hline & & $(1,7)$ & & $(1,7)$ & \\
\hline \multirow[t]{2}{*}{6} & 1378.2 & 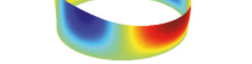 & 1362.4 & $\theta$ & 1.1 \\
\hline & & $(1,2)$ & & $(1,2)$ & \\
\hline \multirow[t]{2}{*}{7} & 1650.8 & Monis & 1644 & (not) & 0.4 \\
\hline & & $(1,8)$ & & $(1,8)$ & \\
\hline \multirow[t]{2}{*}{8} & 2021.5 & ingus? & 2011.9 & (Fons? & 0.5 \\
\hline & & $(1,9)$ & & $(1,9)$ & \\
\hline
\end{tabular}

techniques which are suitable for the thin-walled shell with or without hard coating are also proposed.

3.1. Test System of Vibration Characteristics of TCS with Hard Coating. On the one hand, due to light weight, closed modes, low vibration level, and complicated local vibration of TCS, traditional accelerometer will bring added mass and stiffness to the tested shell [24], which will severely affect the tested frequency and damping results, so laser Doppler vibrometer is used as noncontact response sensor to measure the vibration and frequency information of the shell. On the other hand, different excitation techniques also will result in test error, so the disadvantages of four common vibration excitation devices are compared in Table 4; combining the proposed test method in [25], vibration shaker is finally chosen as excitation source with excitation level being precisely controlled while hammer is used to roughly get natural frequency (which will be described in Section 3.2) by experimental modal test technique. Figure 4 gives the schematic of test system of vibration characteristics of TCS with or without hard coating. The instruments used in the test are as follows: (I) Polytec PDV-100 laser Doppler vibrometer; (II) King-design EM$1000 \mathrm{~F}$ vibration shaker systems; (III) PCB 086C01 modal hammer; (IV) LongWei PS-305DM DC power supply; (V) Aslong JGA25 DC geared motor; (VI) $45^{\circ}$ rotation mirror and $45^{\circ}$ fixed mirror; (VII) LMS SCADAS Mobile Front-End and Dell notebook computer.

In these devices, LMS SCADAS Mobile Front-End and Dell notebook computer are responsible for recording and saving response signal from laser Doppler vibrometer. Dell notebook computer with Intel Core i7 $2.93 \mathrm{GHz}$ processor and $4 \mathrm{G}$ RAM is used to operate LMS Test. Lab 12A software and store measured data. For the natural frequency test, sine sweep excitation is conducted with a closed loop control via accelerometer on the countertop of the vibration shaker, and each natural frequency of TCS with or without hard coating can be precisely determined through each resonant peak in frequency domain. For the damping ratio test, 
TABLE 4: The disadvantages of different vibration excitation devices for vibration test of TCS with or without hard coating.

\begin{tabular}{|c|c|c|c|c|c|}
\hline \multirow[b]{2}{*}{ Excitation device } & \multicolumn{4}{|c|}{ Vibration parameters of TCS } & \multirow[b]{2}{*}{ Disadvantage } \\
\hline & $\begin{array}{c}\text { Natural } \\
\text { frequency }\end{array}$ & Modal shape & Damping ratio & $\begin{array}{l}\text { Vibration } \\
\text { response }\end{array}$ & \\
\hline Hammer & $\sqrt{ }$ & $\sqrt{ }$ & $\times$ & $\times$ & $\begin{array}{l}\text { Pulse excitation level cannot be } \\
\text { precisely controlled and the } \\
\text { excitation force varies for each } \\
\text { measurement, and double hit often } \\
\text { leads to test errors in damping and } \\
\text { vibration response measurement. }\end{array}$ \\
\hline Electromagnetic exciter & $\times$ & $x$ & $\times$ & $\times$ & $\begin{array}{c}\text { The related force sensor will bring } \\
\text { added mass and stiffness to TCS, } \\
\text { which will severely affect test results } \\
\text { of damping, natural frequency, and } \\
\text { so forth. }\end{array}$ \\
\hline Piezoelectric ceramic exciter & $\sqrt{ }$ & $\sqrt{ }$ & $\times$ & $\times$ & $\begin{array}{l}\text { The excitation energy of } \\
\text { piezoelectric ceramic exciter is often } \\
\text { insufficient for TCS, which will } \\
\text { result in poor response signal with } \\
\text { low level of signal noise ratio. }\end{array}$ \\
\hline Vibration shaker & $\sqrt{ }$ & $\sqrt{ }$ & $\sqrt{ }$ & $\sqrt{ }$ & $\begin{array}{l}\text { Excitation frequencies are not that } \\
\text { high, often limited to } 1 \mathrm{~Hz} \sim 3000 \mathrm{~Hz} \text {, } \\
\text { and the test procedures are often } \\
\text { complicated. }\end{array}$ \\
\hline
\end{tabular}
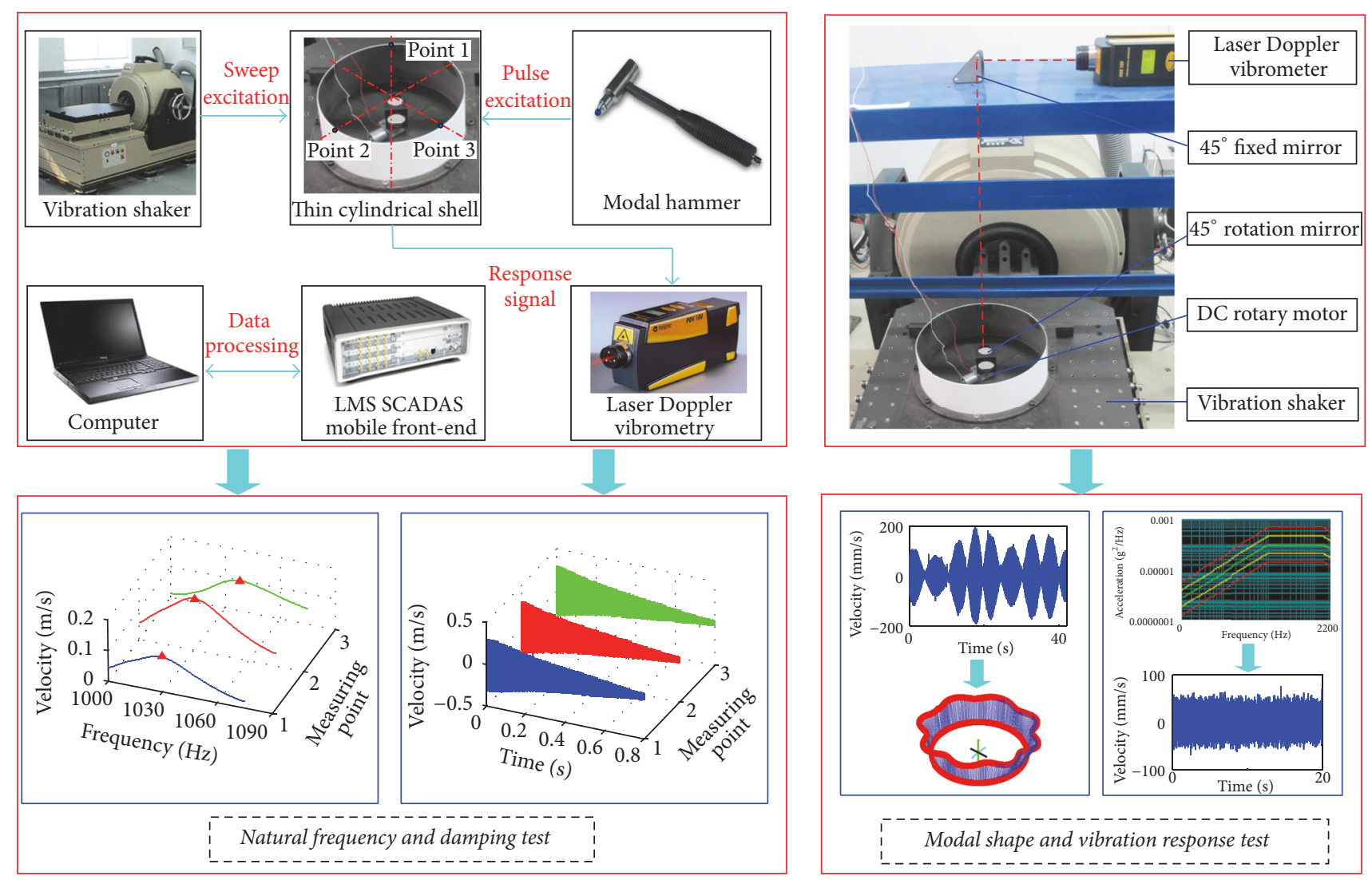

FIGURE 4: The schematic of test system of vibration characteristics of TCS with or without hard coating. 
the sliding-envelope method in time domain is employed, and through fitting the attenuation envelope under different sliding-windows, we can identify each damping ratio of TCS from the corresponding attenuation signal of each mode. For the modal shape test, $45^{\circ}$ fixed mirror is used to change the light path of laser Doppler vibrometer, and DC rotary motor is to drive the $45^{\circ}$ rotation mirror to complete a set of crosssectional scan with $360^{\circ}$ circumferential coverage for the tested shell, so that modal shapes data at certain mode can be obtained efficiently. For random vibration response test, the similar test technique is employed to get circumferential scan data of certain sections of the shell, and the corresponding extracting method is proposed to get the response data of the concerned measuring points (we will describe these test methods in detail in Section 3.2).

\subsection{Test Method of Vibration Characteristics of TCS with Hard} Coating. In this section, on the basis of considering nonlinear stiffness and damping properties of hard coating, the experimental test is divided into two phases, that is, Phase I: no coating TCS measurement and Phase II: coated TCS measurement, and the corresponding test methods and identification techniques which are suitable for the thin-walled shell with or without hard coating are also proposed, as seen in the following test procedures.

\subsubsection{Test Method for Phase I}

(1) Determine Tightening Torque of TCS under Constraint Boundary in the Preexperiment. Because natural frequencies, modal shapes, damping ratios, and vibration responses of TCS with or without hard coating are closely related to constraint boundary, in actual test, we must ensure that one end of the tested shell is effectively clamped; to this end, a torque wrench is used to determine the level of tightening torque on the M8 bolts of clamping-ring, as seen in Figure 1, and the "preexperiment" is adopted to determine the required tightening torque as well as to verify whether or not the tested shell is under fixed constraint boundary. For instance, it should be done at least three times to test natural frequencies, and every time the same level of torque value should be applied on M8 bolts. If test results of the first 3 natural frequencies under three preexperiments are close to each other (for example $1 \sim 3 \mathrm{~Hz}$ ), we will regard this torque value as the determined tightening torque under fixed constraint boundary. If the differences between each natural frequency are big, more than $5 \sim 20 \mathrm{~Hz}$, we need to increase torque value and to repeat preexperiments several times.

(2) Determine the Numbers and Positions of Vibration Measuring Points. Due to the complicated vibrational properties of TCS, such as the very closed modes, the small vibration levels, and abundant local vibration, it is necessary to select multiple measuring points to finish the experiment. (If only one measuring point is chosen, it is very likely to be assigned to the node or nodal line of the shell, and this will severely affect frequency, damping, and response test results.) In this research, by considering test efficiency and precision at the same time, we finally use point 1 , point 2 , and point 3 to get response signal, as seen in Figure 4, which are $120^{\circ}$ with each other and in the same cross section of the shell.

(3) Roughly Measure Each Natural Frequency of TCS without Hard Coating. This step is mainly used to determine the sweep frequency range for step (4), which is often about $85 \% \sim 115 \%$ of each natural frequency. We can roughly get frequency result at a fast sweep rate provided by vibration shaker, or we can use hammer excitation or the theoretical calculated method. No matter what technological skills are used, it is suggested to compare with the resulting frequencies so as to prevent mode missed phenomenon.

(4) Accurately Measure Each Natural Frequency of TCS without Hard Coating. Based on the rough frequency results as well as sweep frequency ranges obtained in step (3), we can use sine sweep excitation technique provided by vibration shaker to get each sweep signal data, which would contain each natural frequency of the shell. Then, employ the smallsegment FFT processing technique to accurately identify natural frequency of each mode. Because natural frequency would be affected if the sweep rate is too fast, it is suggested that the sweep rate meets the relation in (27) proposed by Torvik [26], and he also points out that this sweep rate should always be validated by a comparison with results obtained at an even slower rate:

$$
S<2 \pi \xi_{i}^{2} \omega_{i}^{2}
$$

where $S$ is the sweep rate used in sine sweep test, expressed in $\mathrm{Hz} / \mathrm{s} ; \omega_{i}, \xi_{i}$ are the $i$ th natural frequency and modal damping ratio, respectively.

The time domain signal involving the 5th natural frequency of the tested shell is showed in Figure 5(a). If FFT processing technique is directly applied on this sweep signal, we can obtain its frequency spectrum, as seen in Figure 5(b), and the frequency of the response peak is $1293.5 \mathrm{~Hz}$. However, if the whole time of sweep signal can be divided into small segments and we conduct FFT on each segment of them (in this example, it is $1 \mathrm{~s}$ with respect to the whole time of $40 \mathrm{~s}$ ), the resulting frequency spectrum, as seen in Figure 5(c), is plotted through the combination of the response peaks of each segment (also treated with interpolation and smoothing); the frequency value related to the peak is $1292.0 \mathrm{~Hz}$, which is truly accurate result of the 5th natural frequency. Therefore, for time-dependent sweep signal of TCS, it is necessary to use the small-segment FFT processing technique to accurately get frequency results.

(5) Measure Each Damping Ratio of TCS by Sliding-Envelope Method. In the practical damping measurement, it is found that the half power bandwidth method can be a problem when the test object is the shell structure with much closed mode. For example, when the distance of response peaks between the two modes is too close, it is hard to find frequency values related to half power bandwidth points and then affect the accuracy of identifying damping [27]. Therefore, sliding-envelope method is proposed to test modal damping of TCS coated with or without hard coating [28]; this test technique would firstly excite the structure system 


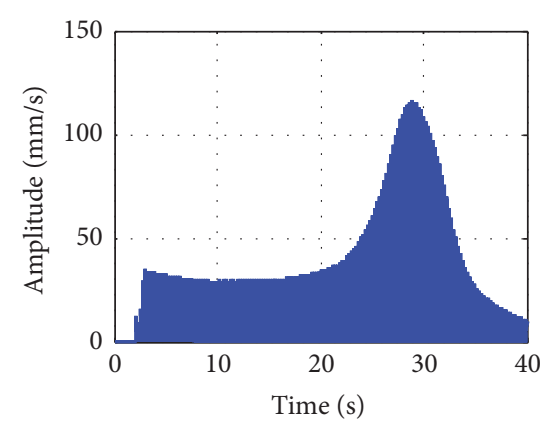

(a) The raw sweep signal

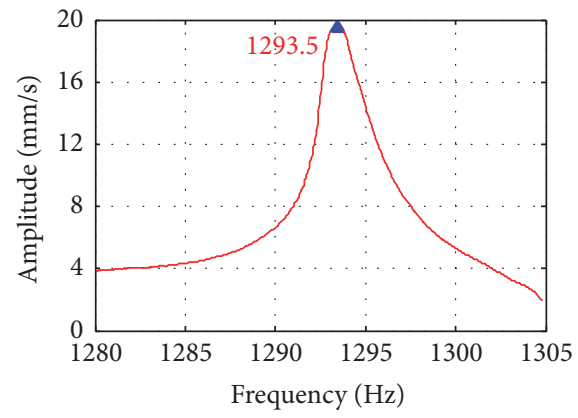

(b) The spectrum by direct FFT

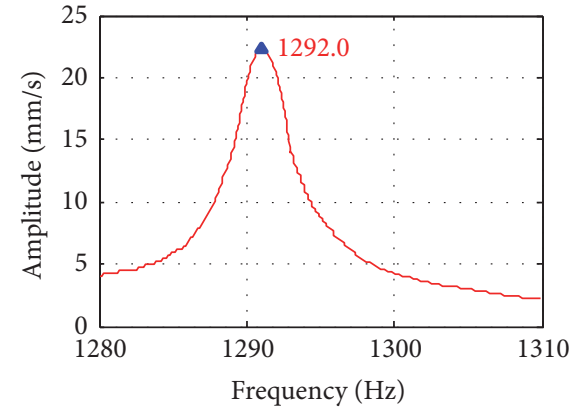

(c) The spectrum by small-segment FFT

FIGURE 5: The 5th natural frequency of TCS obtained by different FFT processing techniques.

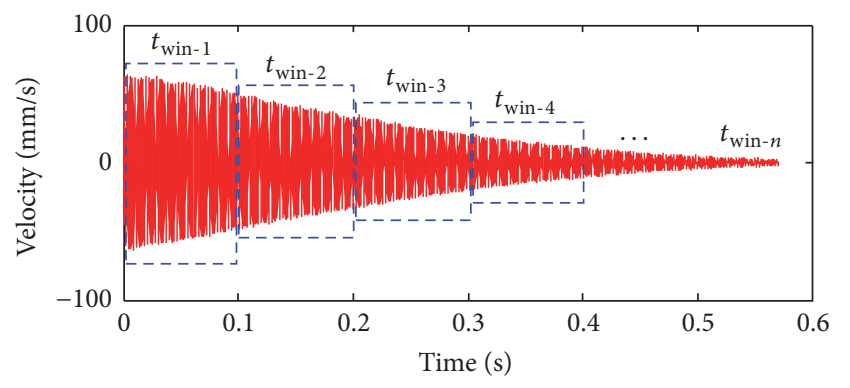

Figure 6: The 3rd time-attenuation signal of TCS without hard coating obtained by sliding-envelope method.

to reach resonance state corresponding to a certain mode, whose natural frequency is already obtained in step (4). Then, after the resonance response is stable, we cut the excitation energy and record free attenuation signal at the same time. Finally, the modal damping ratio of the cylindrical shell structure can be identified with high accuracy by sliding-envelope method, which also contains the following data processing techniques, such as filtering, sliding-window processing, and envelope-fitting. Figure 6 gives the $3 \mathrm{rd}$ attenuation signal at measuring point 1 of TCS obtained by laser Doppler vibrometer; consequently the corresponding damping results can be obtained based the Matlab program. In the data processing we totally divide such attenuation signal into 6 slidingwindows and each time length of sliding-window is $0.1 \mathrm{~s}$, and Figure 7 shows the parts of the attenuation envelope-curves of the 3rd damping obtained by sliding-envelope method.

(6) Measure Each Modal Shape of TCS by Laser Rotating Scanning Method. Although experimental modal method can still be a frequently used technology to get modal shape of TCS, it often needs to constantly move the positions of the excitation or response points, which would lead to the problem of lower efficiency. Meanwhile, due to the uneven and insufficient excitation energy distributed on the TCS, the resulting modal shapes are sometimes dubious if the excitation points determined by this method are too few. In order to solve these problems, laser rotating scanning method is proposed to measure modal shapes of TCS coated with or without hard coating [29]; this test technique would firstly employ one of natural frequencies of TCS obtained in step (4) to drive the shell under the resonance state. Then, use $45^{\circ}$ rotation mirror to complete $360^{\circ}$ circumferential scan of one or two cross sections of the shell. Finally, load the scan signals manipulated after data-reduction process into the established experimental model; the modal shapes at certain mode can be drawn. Repeating the above substeps, we can finish the drawing of shapes results at other modes with higher efficiency and accuracy than the traditional shape test methods.

(7) Measure Random Vibration Response of TCS by Laser Rotating Scanning Method. In the natural world, the random vibration environment is quite common, and vibration fatigues and damage of TCS are often induced by the random dynamic load. Therefore, it is of great importance in the academic and engineering fields to evaluate the random vibration response characteristics of TCS coated with or without hard coating. In this step, circumferential scan data of certain sections of the shell is firstly obtained based on the laser rotating scanning method, and consequently the following extracting method is proposed to get response data of TCS under random excitation.

Assuming $n$ represents the total number of the measuring points of TCS, which can meet a complete circumferential scan (usually $n \geq 360$ ), $t_{0}=0$ represents the starting time corresponding to the first measuring point, and $t_{\text {com }}$ represents the completed time of such circumferential scan, then the time gap $\tau$ between the two adjacent measuring points can be expressed as

$$
\tau=\frac{t_{\mathrm{com}}}{(n-1)}
$$

At this moment, the concerned scanning time $t_{k}$ corresponding to the $k$ th measuring points can be expressed as

$$
t_{k}=t_{0}+\tau(k-1)=t_{0}+\frac{t_{\mathrm{com}}}{(n-1)}(k-1)
$$




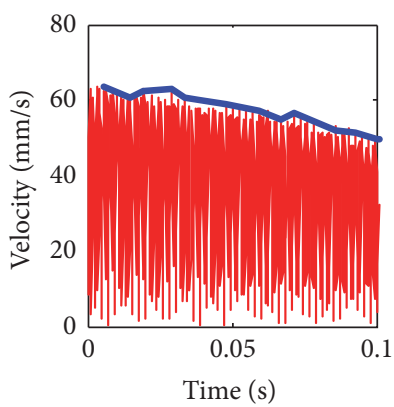

(a) Window 1

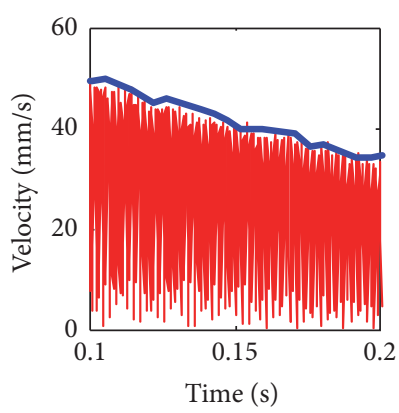

(b) Window 2

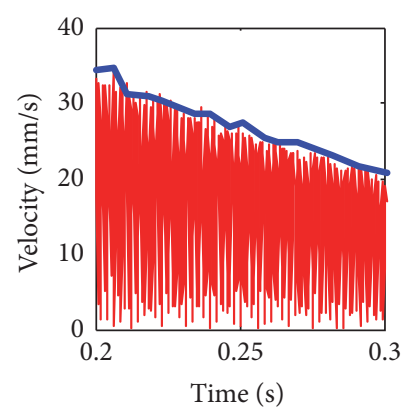

(c) Window 3

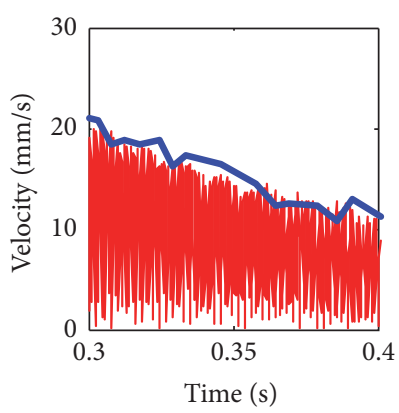

(d) Window 4

FIgURE 7: Attenuation envelope-curves of the 3rd damping under different sliding-windows obtained by sliding-envelope method.

TABLE 5: The setting parameters used in random vibration response measurement when the total root mean square value is $0.45 \mathrm{~g}$.

\begin{tabular}{lcccccccc}
\hline Order & $\begin{array}{c}\text { Frequency } \\
(\mathrm{Hz})\end{array}$ & $\begin{array}{c}\text { Left slope } \\
(\mathrm{dB} / \mathrm{Oct})\end{array}$ & $\begin{array}{c}\text { Acceleration } \\
\left(\mathrm{g}^{2} / \mathrm{Hz}\right)\end{array}$ & $\begin{array}{c}\text { Right slope } \\
(\mathrm{dB} / \mathrm{Oct})\end{array}$ & $\begin{array}{c}\text { High-abort } \\
(\mathrm{dB})\end{array}$ & $\begin{array}{c}\text { Hi-alarm }(\mathrm{dB}) \\
(\mathrm{dB})\end{array}$ & $\begin{array}{c}\text { Low-alarm } \\
(\mathrm{dB})\end{array}$ & $\begin{array}{c}\text { Low-abort } \\
(\mathrm{dB})\end{array}$ \\
\hline 1 & 1 & & & 3 & 6 & 3 & -3 & -6 \\
2 & 100 & 3 & 0.0001 & & 6 & 3 & -3 & -6 \\
3 & 1500 & & 0.0001 & -3 & 6 & 3 & -3 & -3 \\
4 & 2200 & -3 & & & 6 & 3 & -6 \\
\hline
\end{tabular}

Then, the time width criterion which is mainly used for the extraction operation of laser scanning data can be established and expressed as

$$
\Delta t \leq \frac{d}{v}
$$

where $\Delta t$ is the time width, $d$ is the diameter of laser point with the size of $1 \mathrm{~mm} \sim 5 \mathrm{~mm}$, and $v$ is the scanning rate of DC rotary motor with the range of $1 \mathrm{r} / \mathrm{min} \sim 2 \mathrm{r} / \mathrm{min}$. It should be noted that usually the smaller the time width $\Delta t$, the more accurate the laser scanning data that can we get, but for the sake of convenient extraction, we also find out that $\Delta t$ should not be less than $0.01 \mathrm{~s}$; otherwise it will lead to much larger sampling frequency in the measurement, and that will occupy a large amount of disk space.

Finally, based on the time width criterion, we can extract vibration response data corresponding to the different measuring points. For example, for $k=1$, that is, the 1st measuring points, we can use scanning time of $t_{0} \sim t_{0}+\Delta t$ as the time width. For $k=2, \ldots, n-1$, that is, from the 2 nd measuring points to the $n-1$ th measuring points, we can extract vibration response data from the scanning time of $t_{k}-0.5 \Delta t \sim$ $t_{k}+0.5 \Delta t$. For $k=n$, that is, the $n$th measuring points, the scanning time of $t_{\text {com }}-\Delta t \sim t_{\text {com }}$ would be used as the time width for extraction operation.

In the measurement, we use the following random excitation spectrum, as seen in Figure 8, whose total root mean square value is $0.45 \mathrm{~g}$ and its setting parameters are listed in Table 5, and combine the self-designed Matlab program with the principle illustrated from (28) to (30); we can finally get random vibration response results of no coating TCS.

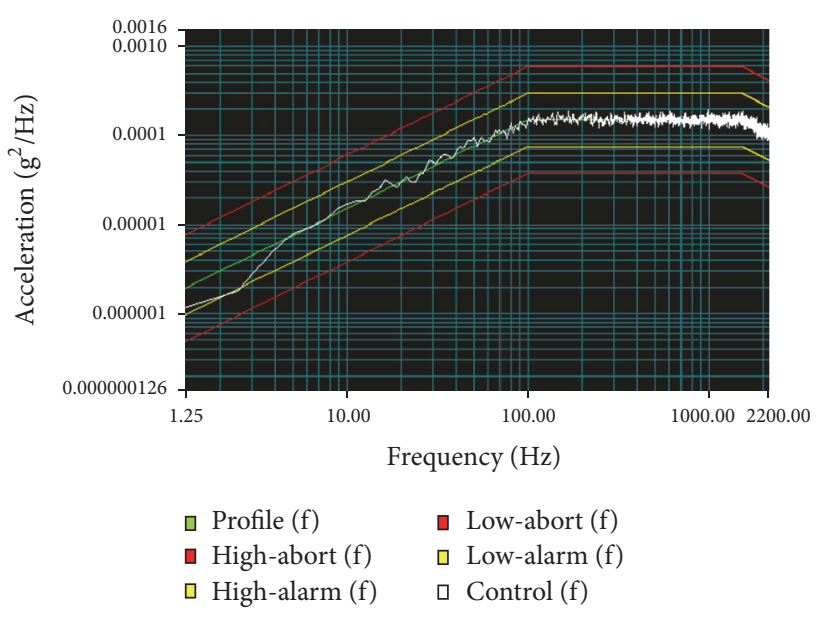

FIGURE 8: Random excitation spectrum used in random vibration response measurement.

Consequently, extract the time waveforms and power spectral density (PSD) functions at the measuring point 1 , point 2 , and point 3; by comparing with the corresponding results of coated TCS, we can know exactly whether or not the hard coating would reduce vibration of TCS.

\subsubsection{Test Method for Phase II}

(1) Determine Constraint Boundary of Coated TCS by the Same Torque Tightening Used in Phase I and Measure the Vibration Parameters at the Same Positions of Measuring Points. In order to ensure that the constraint boundary will not be 
changed when the TCS is unloaded from the clamping-ring for coating treatment, we must use the same torque tightening used in Phase I to effectively tighten eight M8 bolts of TCS with hard coating. Then, at the same positions of measuring points, we can carry out vibration parameters measurement, including natural frequencies, modal shapes, damping ratios, and vibration responses, so that the effect degree and trend of hard coating on the shell structure can be discovered.

(2) Accurately Measure Each Natural Frequency of Coated TCS under Different Excitation Levels. Good practice in our test series dictates that natural frequency of TCS with hard coating will decrease as the external excitation level increases, indicating some characteristics of "softened stiffness." If the test is carried out from the traditional sweep direction, that is, from low frequency to high frequency, the nonlinear jump phenomenon is very likely to occur, which will heavily affect the precise measurement of natural frequencies. Therefore, the test should be done from sweep direction of high frequency to low frequency. Besides, because sweep frequency ranges are already determined in step (3) of Phase I (when the shell is coated with hard coating, the changes of natural frequencies will always be there, but its varied degrees cannot exceed such sweep frequency range), so we only need to adjust the excitation levels and then employ the smallsegment FFT processing technique to accurately measure each natural frequency of coated TCS.

(3) Measure Each Damping Ratio of Coated TCS under Different Excitation Levels. In the study on vibration measurement techniques on TCS, it is also found that damping parameters will change with the external excitation levels, and if the thickness of hard coating is larger, then the nonlinear damping influence would be more obvious. Therefore, similar to step (5) in Phase I, we firstly use the resulting natural frequencies obtained under different excitation levels in step (2) to excite the coated shell system to reach resonance state, and then based on the free attenuation signal, we can employ the sliding-window envelope method to identify damping results under different excitation levels.

(4) Measure Each Modal Shape of Coated TCS under Different Excitation Levels. According to the related literature [30, 31], hard coating material not only will affect natural frequency of TCS, but also may lead to the change of modal shape. Therefore, similar to step (6) in Phase I, we firstly use the resulting natural frequencies obtained under different excitation levels in step (2) to excite the coated shell system to reach resonance state, and then we can employ laser rotating scanning method to measure each modal shape of coated TCS under different excitation levels.

(5) Measure Random Vibration Response of Coated TCS under the Same Random Excitation Level Used in Phase I. In this step, employing the same random excitation level used in Phase I, we can measure random vibration response of TCS with hard coating by laser rotating scanning method. Then, based on the established time width criterion, we can extract time waveforms and spectrums at the same positions of measuring points. By comparing with the no coating results, we can objectively evaluate vibration reduction effect of hard coating coated on the shell structure.

\section{Test Results of Vibration Parameters of TCS Coated with and without Hard Coating}

In this section, based on the established experiment system in Section 3.1, natural frequencies, modal shapes, damping ratios, and vibration responses of TCS coated with and without hard coating are obtained by the proposed test method.

Firstly in Phase I when the TCS is not coated with hard coating, several tightening torques are chosen to tighten eight M8 bolts in the preexperiment, and after the repeated comparisons and experiments, the torque value of $50 \mathrm{Nm}$ is finally determined. Then, when the tested shell is well constrained, we use point 1 , point 2 , and point 3 to get response signal, which are $120^{\circ}$ with each other and in the same cross section of the shell and the axial distance from this section to free end of TCS is about $3 \mathrm{~mm}$. Next, the different sweep rates, such as $1 \mathrm{~Hz} / \mathrm{s}, 2 \mathrm{~Hz} / \mathrm{s}$, and $4 \mathrm{~Hz} / \mathrm{s}$, are compared in the measurement, and the rate of $1 \mathrm{~Hz} / \mathrm{s}$ is finally employed to accurately get natural frequencies by sine sweep excitation technique. At last, by using the sliding-envelope method and laser rotating scanning method, we can obtain frequencies, shapes, damping, and vibration response results of TCS with high accuracy and efficiency.

Then in Phase II when the TCS is coated with hard coating, we use the same torque tightening of $50 \mathrm{Nm}$ to effectively tighten eight M8 bolts of the shell, and with the same test techniques in Phase I, we can get the corresponding vibration parameters at the same positions under different excitation levels, so that we can investigate nonlinear stiffness and damping properties of the shell coated with hard coating.

For Phase I, the following setups and parameters are chosen to get frequency and damping results: (I) the constant excitation level of $1 \mathrm{~g}$; (II) sweep rate of $1 \mathrm{~Hz} / \mathrm{s}$; (III) frequency resolution of $0.5 \mathrm{~Hz}$; (IV) Hanning window for sweep response signal with downward sweep direction; (V) frequency range of 0-2048 Hz. Besides, the following parameters are set to get mode shapes and vibration responses: (I) excitation level of $1 \mathrm{~g}$ to get shape data; (II) the total root mean square value of $0.45 \mathrm{~g}$ in the random excitation; (III) rectangular window for stable response signal with Hanning window for random signal; (IV) sampling frequency of $8192 \mathrm{~Hz}$; (V) rotated scan speed of $2 \mathrm{r} / \mathrm{min}$; (VI) random excitation time not less than $10 \mathrm{~s}$.

For Phase II, coated TCS measurement, the setups are basically similar to the above parameters determined in Phase I except for the excitation levels, which need to be changed to measure the nonlinear stiffness and damping properties of the shell coated with hard coating. Because it is hard for vibration shaker to control large amplitude when the excitation frequencies are low (which would lead to overload phenomenon), the lower excitation levels such as $0.5 \mathrm{~g}, 1 \mathrm{~g}, 2 \mathrm{~g}$, $4 \mathrm{~g}$, and $6 \mathrm{~g}$ are chosen to get the first four mode parameters, while for the rest of the modes, the larger excitation levels, such as $1 \mathrm{~g}, 2 \mathrm{~g}, 4 \mathrm{~g}, 8 \mathrm{~g}, 12 \mathrm{~g}$, are used to get the concerned 
results. It should be noted that in the mode shape measurement, each mode shape is assembled from two sets of crosssectional scans, one is in the section which includes point 1 , point 2 , and point 3 , and the other is about $25 \mathrm{~mm}$ to the clamped end of the shell, which is restricted by the height of DC rotary motor itself, but does not affect the test results when the number of axial half-waves $m=1$ (and $n$ represents the number of circumferential waves of TCS). Besides, in order to get good results, some viscoelastic rubber materials are applied to the joint surface between the vibration shaker, DC rotary motor, and the $45^{\circ}$ rotation mirror to absorb and suppress the vibration transmission, but they are only effective when the lower excitation levels (e.g., $0.5 \mathrm{~g}, 1 \mathrm{~g}, 2 \mathrm{~g}$, and $4 \mathrm{~g}$ ) are used, so when the excitation levels become much larger, we cannot use laser rotating scanning method to get satisfactory shape results.

The first four natural frequency, damping, and shape results of cantilever cylindrical shell coated with and without hard coating under different excitation levels are listed in Tables 6,8 , and 10 , while the 5 th to 8 th results are listed in Tables 7, 9, and 11, and in order to clearly describe the effect degree and trend of hard coating, the scattergrams of natural frequencies and damping ratios of the shell coated with and without hard coating under the excitation levels of $1 \mathrm{~g}, 2 \mathrm{~g}$, and $4 \mathrm{~g}$ are also given, as shown in Figures 9 and 10, Besides, Figures 11, 12, 13, and 14 give the random vibration responses results of cantilever cylindrical shell with and without hard coating at 3 measuring points, such as time waveforms and power spectrum density functions.

\section{The Influence Analysis on the Vibration Characteristics of TCS with Hard Coating}

In this section, on the basis of the measured results in Section 4 , the influences of hard coating on natural frequencies, mode shapes, damping ratios, and vibration response of TCS are analyzed and discussed in detail.

(1) The Influence on Natural Frequencies of TCS. From Tables 6 and 7 and Figure 9, the following can be found: (I) For the most modes of TCS under cantilever boundary condition, hard coating will result in the decrease of natural frequencies, but because the coating material is very thin, the decreased level is not very big, which is within the range of $0.1 \% \sim 2.4 \%$, and this experimental conclusion agrees well with the calculated results obtained based on the theoretical model of coated TCS. (II) Hard coating will turn shell structure into a nonlinear system with variable stiffness under different excitation levels, and with the increase of excitation levels, the softened stiffness phenomenon is becoming more obvious. Taking the 2nd and 3rd natural frequencies, for example, they basically decrease from $1.3 \%$ to $2.4 \%$ compared with the ones under no coating state. (III) The increase of excitation level would also result in the decrease of high order natural frequencies, but the decreased degree is very small. Taking the 7th and 8th natural frequencies, for example, the maximum of the decreased frequency results is about $0.2 \% \sim 0.3 \%$ compared with the ones without coating. Therefore, it can be

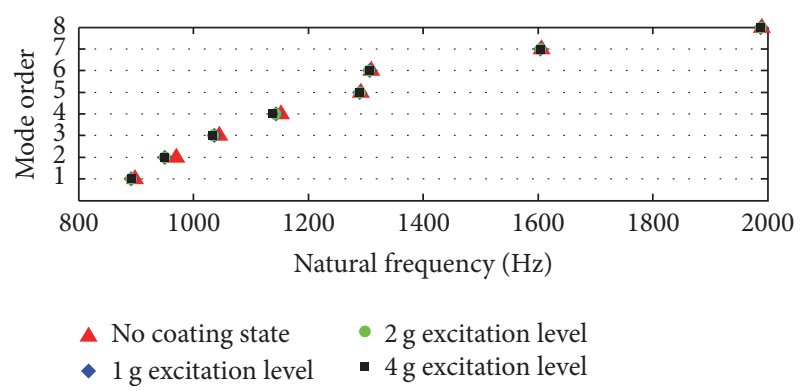

Figure 9: The scattergrams of natural frequencies of cantilever cylindrical shell coated with and without hard coating under different excitation levels.

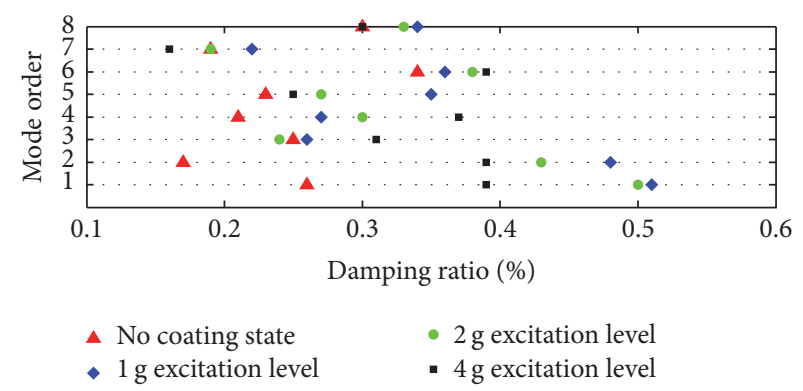

FIGURE 10: The scattergrams of damping ratios of cantilever cylindrical shell coated with and without hard coating under different excitation levels.

concluded that high order natural frequencies are less affected by hard coating than the other frequency results.

Because we have calculated the resulting natural frequencies of TCS under the two coating states by the self-written Matlab program in Section 2.2, here we also compare these calculated frequency results with the measured ones (for the coating state, the results under $1 \mathrm{~g}$ level are used), as seen in the Table 12, and in order to explain them clearly, Figure 15 gives the relation curves of the measured and calculated natural frequencies of cantilever cylindrical shell with and without hard coating. From Tables 3 and 12, we can find out that the theoretically calculated natural frequencies are higher than the measured results, which indeed contain some calculation errors yet are still within the acceptable range of $1.2 \% \sim 5.2 \%$. The reasons for that errors may come from (I) inaccurate material parameters for both the shell and hard coating; (II) the errors of support stiffness in theoretical model; (III) the actual nonlinear stiffness and damping effects between the coating and the metal substrate; (IV) complex boundary conditions of the shell which are difficult to simulate in the theoretical model.

Although the calculated frequency results are inaccurate, we can still make good use of their changing trends. From the relation curves of the measured and calculated frequency results in Figure 15, we can discover that with the increase of modal order of cantilever cylindrical shell hard coating will be less able to influence its natural frequencies. For example, the 7 th and 8 th frequencies results under coating 


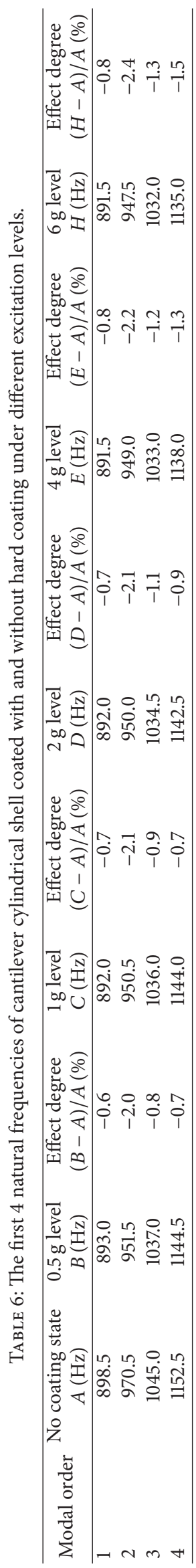




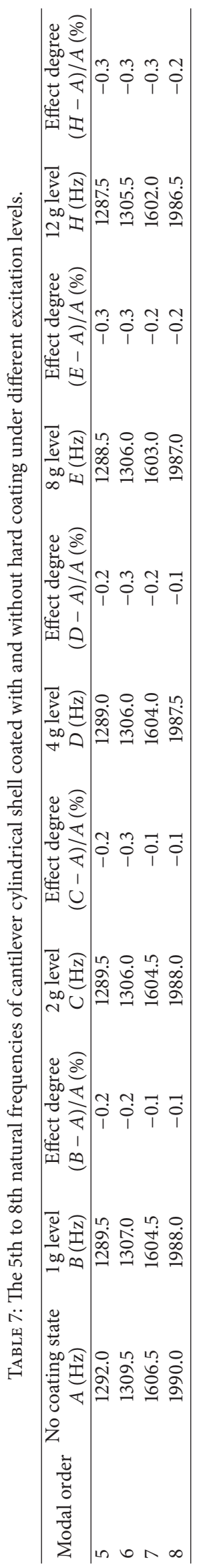




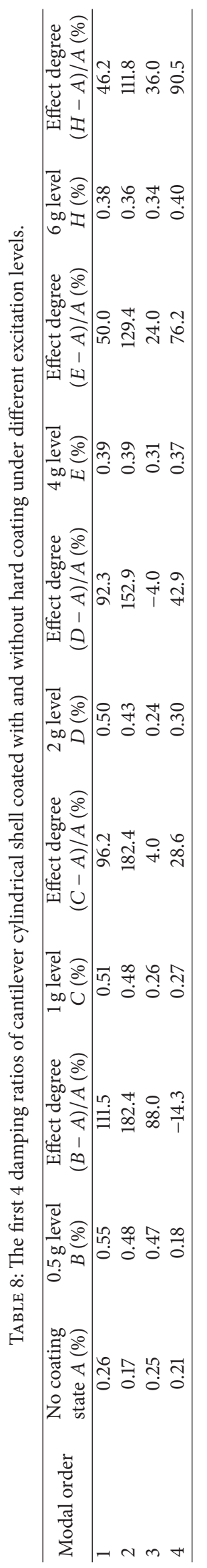




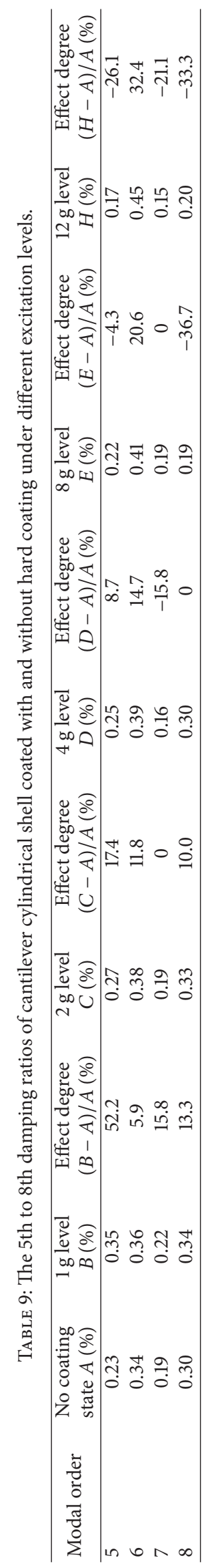


TABLE 10: The first 4 mode shapes of cantilever cylindrical shell coated with and without hard coating under different excitation levels.

\begin{tabular}{|c|c|c|c|c|}
\hline Modal order & $\begin{array}{c}\text { No coating state } \\
\qquad A(m, n)\end{array}$ & $\begin{array}{c}0.5 \mathrm{~g} \text { level } \\
B(m, n)\end{array}$ & $\begin{array}{l}1 \mathrm{~g} \text { level } \\
C(m, n)\end{array}$ & $\begin{array}{l}2 \mathrm{~g} \text { level } \\
D(m, n)\end{array}$ \\
\hline 1 & $(1,5)$ & $(1,5)$ & $(1,5)$ & $(1,5)$ \\
\hline 2 & $(1,4)$ & $(1,4)$ & $(1,4)$ & $(1,4)$ \\
\hline 3 & $(1,6)$ & $(1,6)$ & $(1,6)$ & $(1,6)$ \\
\hline 4 & $(1,3)$ & $(1,3)$ & $(1,3)$ & $(1,3)$ \\
\hline
\end{tabular}

TABLE 11: The 5th to 8th mode shapes of cantilever cylindrical shell coated with and without hard coating under different excitation levels.

\begin{tabular}{|c|c|c|c|c|}
\hline Modal order & $\begin{array}{c}\text { No coating state } \\
A(m, n)\end{array}$ & $\begin{array}{l}1 \mathrm{~g} \text { level } \\
B(m, n)\end{array}$ & $\begin{array}{l}2 \text { g level } \\
C(m, n)\end{array}$ & $\begin{array}{l}4 \text { g level } \\
D(m, n)\end{array}$ \\
\hline 5 & $(1,7)$ & $(1,7)$ & $(1,7)$ & $(1,7)$ \\
\hline 6 & $(1,2)$ & $(1,2)$ & $(1,2)$ & $(1,2)$ \\
\hline 7 & $(1,8)$ & $(1,8)$ & $(1,8)$ & $(1,8)$ \\
\hline 8 & $(1,9)$ & $(1,9)$ & $(1,9)$ & $(1,9)$ \\
\hline
\end{tabular}

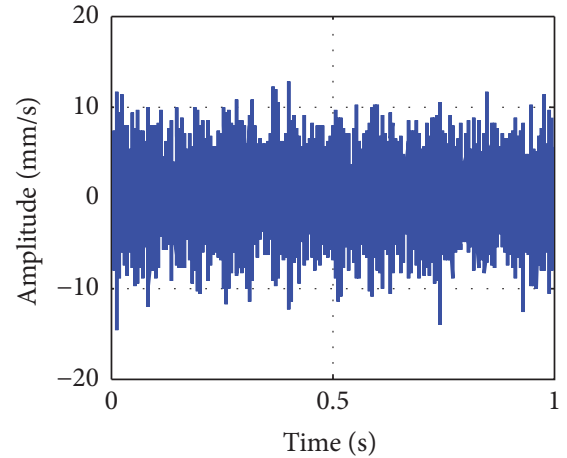

(a) Point 1

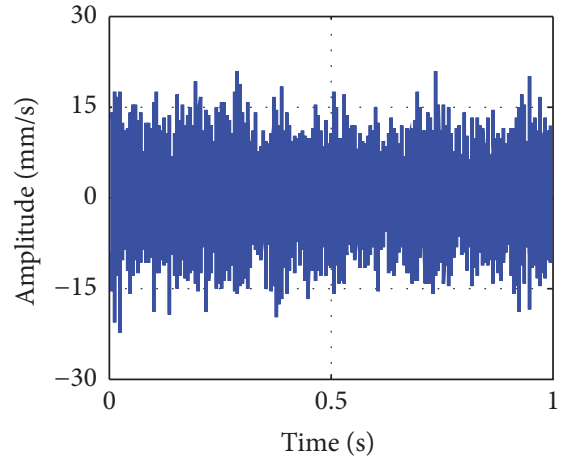

(b) Point 2

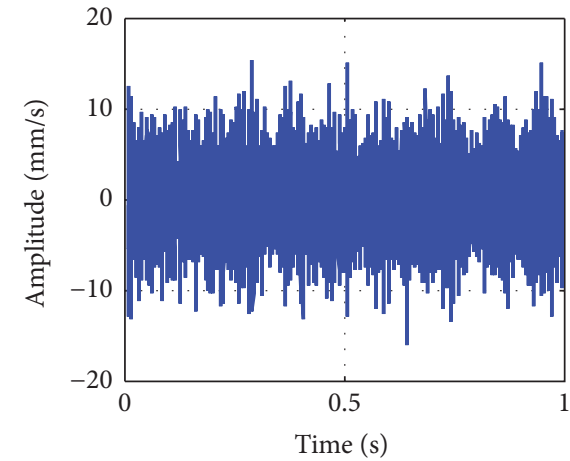

(c) Point 3

FIGURE 11: The measured time waveforms of cantilever cylindrical shell without hard coating at 3 measuring points. 


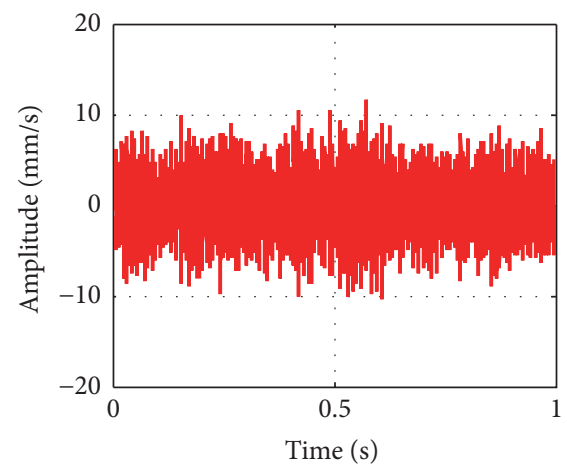

(a) Point 1

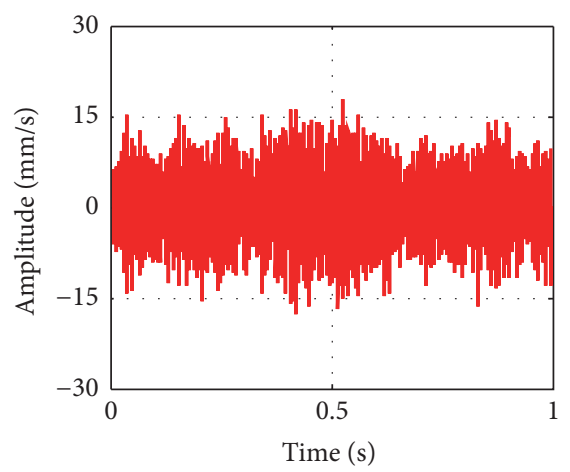

(b) Point 2

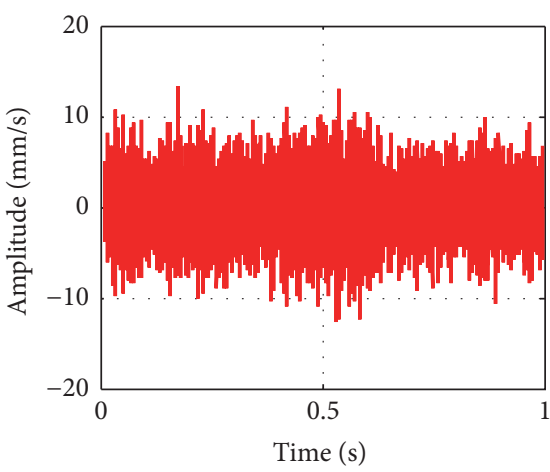

(c) Point 3

FIGURE 12: The measured time waveforms of cantilever cylindrical shell with hard coating at 3 measuring points.

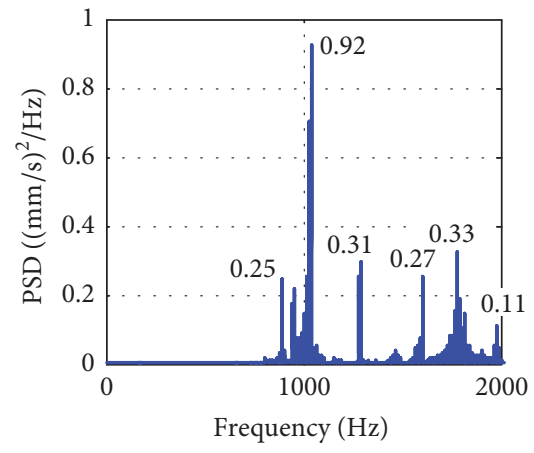

(a) Point 1

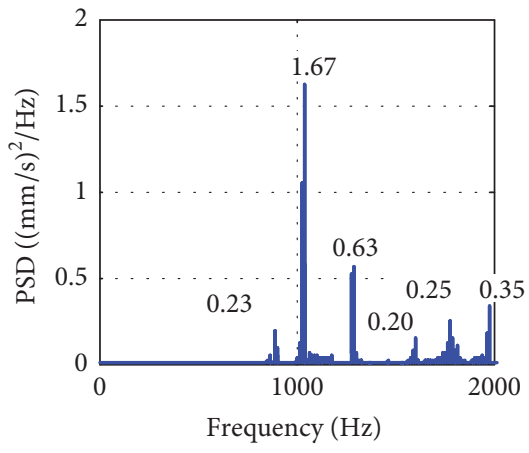

(b) Point 2

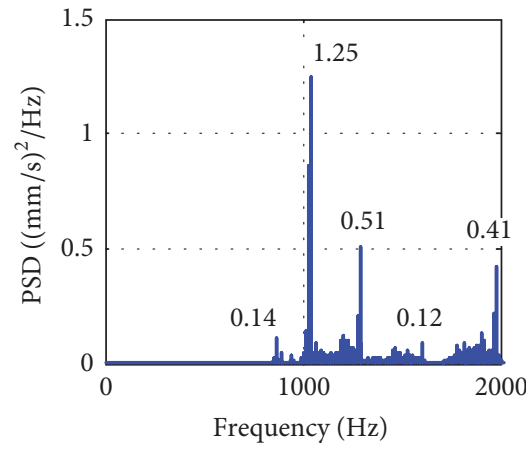

(c) Point 3

Figure 13: The measured PSD functions of cantilever cylindrical shell without hard coating at 3 measuring points.

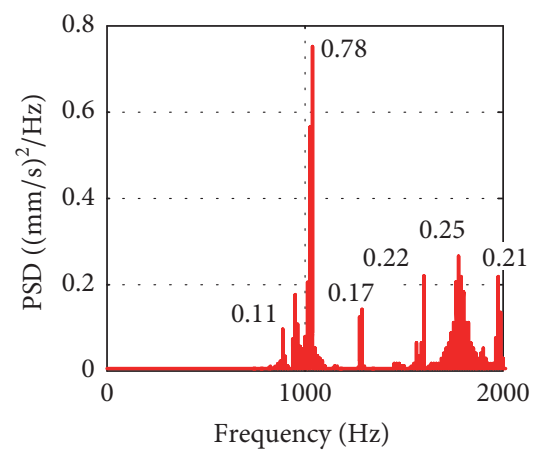

(a) Point 1

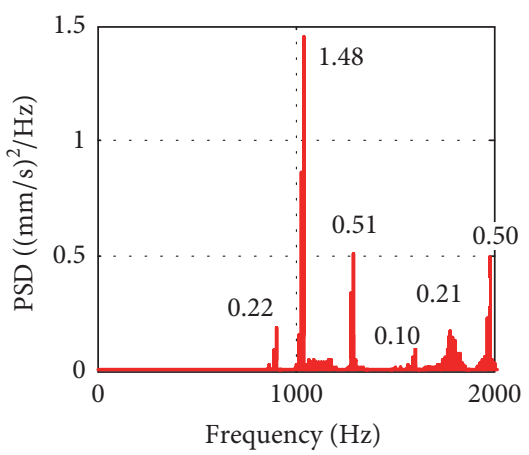

(b) Point 2

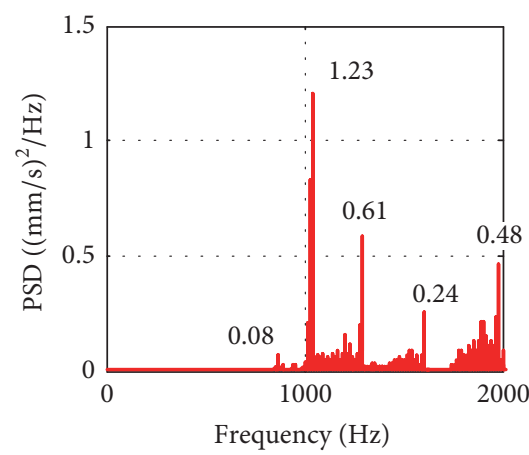

(c) Point 3

FIGURE 14: The measured PSD functions of cantilever cylindrical shell with hard coating at 3 measuring points.

or no coating states are close to each other, which show the resulting frequencies are less affected by hard coating.

(2) The Influence on Damping Ratios of TCS. From Tables 8 and 9 and Figure 10, the following can be found: (I) Hard coating will turn shell structure into a nonlinear system with variable damping under different excitation levels, and for some modes of TCS, the damping ratios damping will be increased obviously. For example, the 1st and 2nd damping results under the excitation level of $1 \mathrm{~g}$ can increase from
$111.5 \%$ to $182.4 \%$ compared with the ones under no coating state, but contrary to our expectations, the damping effects of coated TCS are not proportional to the increased excitation levels; for example, the damping of the 1st, the 2nd, and the 5 th mode is inclined to fall instead of increase. (II) For most modes of TCS, its damping will be increased by hard coating. However, for a small part of modes of TCS, the damping results will be decreased compared with the ones under no coating state, and the decreased degrees are within the range of $4 \% \sim 36.7 \%$. (III) There seem to be some 
TABLE 12: The calculated natural frequencies and mode shapes of cantilever cylindrical shell with and without hard coating and their errors.

\begin{tabular}{|c|c|c|c|c|c|c|}
\hline \multirow[b]{2}{*}{ Modal order } & \multicolumn{3}{|c|}{ No coating state } & \multicolumn{3}{|c|}{ Coating state } \\
\hline & Measured $A(\mathrm{~Hz})$ & Calculated $B(\mathrm{~Hz})$ & $\begin{array}{c}\text { Errors } \\
(B-A) / A(\%)\end{array}$ & Measured $A(\mathrm{~Hz})$ & Calculated $B(\mathrm{~Hz})$ & $\begin{array}{c}\text { Errors } \\
(B-A) / A(\%)\end{array}$ \\
\hline 1 & 898.5 & 923.1 & 2.7 & 893.0 & 918.4 & 2.8 \\
\hline 2 & 970.5 & 985.3 & 1.5 & 951.5 & 972.3 & 2.2 \\
\hline 3 & 1045.0 & 1064.9 & 1.9 & 1037.0 & 1057.8 & 2.0 \\
\hline 4 & 1152.5 & 1194.6 & 3.7 & 1144.5 & 1171.8 & 2.4 \\
\hline 5 & 1292.0 & 1326.5 & 2.7 & 1289.5 & 1306.3 & 1.3 \\
\hline 6 & 1309.5 & 1378.2 & 5.2 & 1307.0 & 1362.4 & 4.2 \\
\hline 7 & 1606.5 & 1650.8 & 2.8 & 1604.5 & 1644 & 2.5 \\
\hline 8 & 1990.0 & 2021.5 & 1.6 & 1988.0 & 2011.9 & 1.2 \\
\hline
\end{tabular}

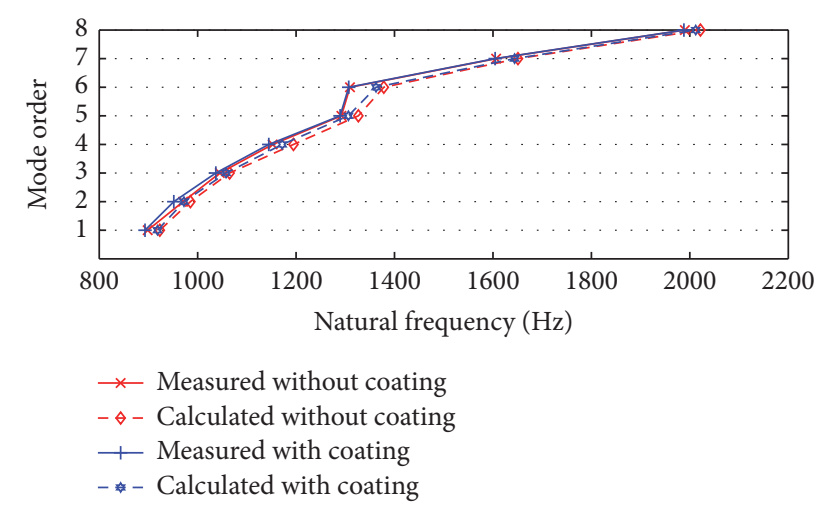

FIGURE 15: The relation curves of the measured and calculated natural frequencies of cantilever cylindrical shell with and without hard coating.

uncertainties about the damping effects of coated TCS, and with the increase of excitation levels, damping effects are becoming weak, especially for high order damping ratios of the shell, such as the 7 th and the 8 th damping results; the largest decreased degree can reach $21.1 \% \sim 36.7 \%$. It should be noted that there might exist some inaccuracies in the damping results due to the influence of constraint boundary of the shell as well as the complexity of damping mechanism of the shell itself, but the above conclusions remind us that in order to make better use of this coating material, we must carefully choose the concerned antivibration frequency range of the shell; otherwise it may lead to some negative effects.

(3) The Influence on Mode Shapes of TCS. From Tables 10 and 11, the following can be found: (I) When the thickness of hard coating is less than $0.31 \mathrm{~mm}$ (at this time, the related thickness ratio between the coating and the substrate is about 0.16 ), although different excitation levels are used in the mode shapes test, the shape results of the shell with hard coating cannot be easily changed. (II) According to the test experience, it is very likely that the shape results of cantilever cylindrical shell with hard coating will eventually be changed once the thickness of hard coating or the thickness ratio rises beyond a certain value, and thus the variations of frequency and damping results of TCS with hard coating will become more complicated.

(4) The Influence on Vibration Response of TCS. From Figures $11,12,13$, and 14, the following can be found: (I) Comparing the measured time waveforms at three measuring points under the same random excitation level, most of the time the amplitudes of the coated TCS are relatively lower than that of TCS without hard coating, and the time domain results can preliminarily demonstrate the effectiveness of vibration reduction of hard coating material. (II) Comparing the measured PSD functions at three measuring points under the same random excitation, the response peaks of the coated TCS are also lower than that of TCS without hard coating in most of the frequency points, so it can be further confirmed that hard coating can indeed play an important role in vibration reduction. However, in the frequency range of $1500 \mathrm{~Hz} 2000 \mathrm{~Hz}$, especially near the frequency of $2000 \mathrm{~Hz}$, the response peaks of the coated TCS become larger than the ones without coating, and combining the measured high order damping ratios, such as the 7th and the 8th damping results, it can be found out that the damping effects of hard coating on the higher frequency range of the shell are weak and ineffective.

\section{Conclusions}

This research has investigated the influence on vibration characteristics of cantilever cylindrical shell with hard coating experimentally, and the theoretical model of TCS with hard coating is also established to roughly master vibration characteristics of shell structure. Based on the calculated and experimental results, the following conclusions can be drawn:

(1) Hard coating will turn shell structure into a nonlinear system with variable stiffness and damping under different excitation levels, and for the most modes of cantilever cylindrical shell, hard coating will result in the decrease of natural frequencies, but the decreased level is not very big, which is within the range of $0.1 \%$ $2.4 \%$. Beside, as the hard coating material is very thin, the mode shapes of the shell cannot be easily changed by such coating. 
(2) For some modes of cantilever cylindrical shell, hard coating will increase their damping obviously, while for a small part of modes of the shell, hard coating will decrease the damping results, which is within the range of $4 \% \sim 36.7 \%$. Besides, with the increase of excitation levels, damping effects of hard coating are becoming weak, especially for high order damping ratios of the shell.

(3) Hard coating can play an important role in vibration reduction of cantilever cylindrical shell based on the measured random vibration response data in time and frequency domain, respectively. But it is found out that its damping effects on the higher frequency range of the shell are weak and ineffective. Therefore, in order to make better use of this coating material, we must carefully choose the concerned antivibration frequency range of the shell; otherwise it may lead to some negative effects.

\section{Appendix}

$$
\begin{aligned}
k_{i j}^{11} & =\frac{\pi L}{2 R} \int_{0}^{1}\left[n^{2} A_{66} P_{i}(\kappa) P_{j}(\kappa)+\frac{R^{2} A_{11}}{L^{2}} \dot{P}_{i}(\kappa)\right. \\
\cdot & \left.\dot{P}_{j}(\kappa)\right] d \kappa \\
k_{i j}^{12} & =\frac{\pi L}{2 R} \int_{0}^{1}\left[\frac{n}{L}\left(R A_{12}+B_{12}\right) \dot{P}_{i}(\kappa) P_{j}(\kappa)\right. \\
& \left.+\frac{n}{L}\left(R A_{66}+B_{66}\right) P_{i}(\kappa) \dot{P}_{j}(\kappa)\right] d \kappa \\
k_{i j}^{13} & =\frac{\pi L}{2 R} \int_{0}^{1}\left[\frac{\left(n^{2} B_{12}+R A_{12}\right)}{L} \dot{P}_{i}(\kappa) P_{j}(\kappa)\right. \\
& -\frac{2 n^{2} B_{66}}{L}\left(R A_{66}+B_{66}\right) P_{i}(\kappa) \dot{P}_{j}(\kappa)-\frac{R^{2} B_{11}}{L^{3}} \\
& \left.\cdot \dot{P}_{i}(\kappa) \ddot{P}_{j}(\kappa)\right] d \kappa \\
& \left.\cdot \dot{P}_{j}(\kappa)\right] d \kappa \\
k_{i j}^{21} & =\frac{\pi L}{2 R} \int_{0}^{1}\left[\frac{n}{L}\left(R A_{12}+B_{12}\right) P_{i}(\kappa) \dot{P}_{j}(\kappa)\right. \\
& \left.+\frac{n}{L}\left(R A_{66}+B_{66}\right) \dot{P}_{i}(\kappa) P_{j}(\kappa)\right] d \kappa \\
k_{i j}^{22} & =\frac{\pi L}{2 R} \int_{0}^{1}\left[\left(n^{2} A_{22}+\frac{2 n^{2} B_{22}}{R}+\frac{n^{2} D_{22}}{R^{2}}\right) P_{i}(\kappa)\right.
\end{aligned}
$$

$$
\begin{aligned}
& k_{i j}^{23}=\frac{\pi L}{2 R} \int_{0}^{1}\left[\frac{n}{L^{2}}\left(2 R B_{66}+4 D_{66}\right) \dot{P}_{i}(\kappa) \dot{P}_{j}(\kappa)\right. \\
& -\frac{n}{L^{2}}\left(R B_{12}+D_{12}\right) P_{i}(\kappa) \ddot{P}_{j}(\kappa) \\
& +\left(n A_{22}+\frac{n^{3} B_{22}}{R}+\frac{n B_{22}}{R}+\frac{n^{3} D_{22}}{R^{2}}\right) P_{i}(\kappa) \\
& \left.\cdot P_{j}(\kappa)\right] d \kappa \\
& k_{i j}^{31}=\frac{\pi L}{2 R} \int_{0}^{1}\left[\frac{\left(n^{2} B_{12}+R A_{12}\right)}{L} P_{i}(\kappa) \dot{P}_{j}(\kappa)\right. \\
& -\frac{2 n^{2} B_{66}}{L}\left(R A_{66}+B_{66}\right) \dot{P}_{i}(\kappa) P_{j}(\kappa)-\frac{R^{2} B_{11}}{L^{3}} \\
& \text {. } \left.\ddot{P}_{i}(\kappa) \dot{P}_{j}(\kappa)\right] d \kappa \\
& k_{i j}^{32}=\frac{\pi L}{2 R} \int_{0}^{1}\left[\frac{n}{L^{2}}\left(2 R B_{66}+4 D_{66}\right) \dot{P}_{i}(\kappa) \dot{P}_{j}(\kappa)\right. \\
& -\frac{n}{L^{2}}\left(R B_{12}+D_{12}\right) \ddot{P}_{i}(\kappa) P_{j}(\kappa) \\
& +\left(n A_{22}+\frac{n^{3} B_{22}}{R}+\frac{n B_{22}}{R}+\frac{n^{3} D_{22}}{R^{2}}\right) P_{i}(\kappa) \\
& \left.\cdot P_{j}(\kappa)\right] d \kappa \\
& k_{i j}^{33}=\frac{\pi L}{2 R} \int_{0}^{1}\left[\left(A_{22}+\frac{2 n^{2} B_{22}}{R}+\frac{n^{4} D_{22}}{R}\right) P_{i}(\kappa) P_{j}(\kappa)\right. \\
& +\frac{4 n^{2} D_{66}}{L^{2}} \dot{P}_{i}(\kappa) \dot{P}_{j}(\kappa)+\frac{R^{2} D_{66}}{L^{4}} \ddot{P}_{i}(\kappa) \ddot{P}_{j}(\kappa) \\
& -\frac{\left(n^{2} D_{12}+R B_{12}\right)}{L^{2}} \ddot{P}_{i}(\kappa) P_{j}(\kappa)-\frac{\left(n^{2} D_{12}+R B_{12}\right)}{L^{2}} \\
& \left.\cdot P_{i}(\kappa) \ddot{P}_{j}(\kappa)\right] d \kappa \\
& K s_{i j}^{11}=\frac{\pi R}{2} k_{u} P_{i}(0) P_{j}(0) \\
& K s_{i j}^{22}=\frac{\pi R}{2} k_{v} P_{i}(0) P_{j}(0) \\
& K s_{i j}^{33}=\frac{\pi R}{2} k_{v} P_{i}(0) P_{j}(0)+\frac{\pi R}{2 L^{2}} k_{\theta} \dot{P}_{i}(0) \dot{P}_{j}(0) \\
& M_{i j}^{11}=\frac{\rho_{t} \pi R L}{2} \int_{0}^{1} P_{i}(\kappa) P_{j}(\kappa) d \kappa \\
& M_{i j}^{22}=\frac{\rho_{t} \pi R L}{2} \int_{0}^{1} P_{i}(\kappa) P_{j}(\kappa) d \kappa \\
& M_{i j}^{33}=\frac{\rho_{t} \pi R L}{2} \int_{0}^{1} P_{i}(\kappa) P_{j}(\kappa) d \kappa .
\end{aligned}
$$




\section{Conflicts of Interest}

The authors declare that there are no conflicts of interest regarding the publication of this paper.

\section{Acknowledgments}

This study was supported by the National Natural Science Foundation of China Grant no. 51375079, the National Natural Science Foundation of China Grant no. 51505070, and the Fundamental Research Funds for the Central Universities of China Grant nos. N150304011 and N160313002.

\section{References}

[1] Leissa, Vibrations of Shells, Acoustical Society of America, Ohio, USA, 1973.

[2] A. Farshidianfar, M. H. Farshidianfar, M. J. Crocker, and W. O. Smith, "Vibration analysis of long cylindrical shells using acoustical excitation," Journal of Sound and Vibration, vol. 330, no. 14, pp. 3381-3399, 2011.

[3] S. H. Yang and A. W. Wang, "Research survey of dynamic response and sound radiation of the submarine pressure shell in water," Journal of Naval University of Engineering, vol. 13, pp. 104-110, 2001.

[4] L. T. Yan, Aero Gas Turbine Vibration and Vibration Reduction, National Defend Industry Press, Beijing, 1991.

[5] D. Y. Wang, "Rotating machinery rotor stator rubbing vibration," Aeroengine, vol. 2, pp. 37-41, 1998.

[6] A. M. Limarga, T. L. Duong, G. Gregori, and D. R. Clarke, "High-temperature vibration damping of thermal barrier coating materials," Surface and Coatings Technology, vol. 202, no. 4-7, pp. 693-697, 2007.

[7] B. A. Potekhin, S. G. Lukashenko, and S. P. Kochugov, "Effect of plasma coatings on the damping properties of structural steels," Metal Science and Heat Treatment, vol. 42, no. 9-10, pp. 407-410, 2000.

[8] E. Stoker, S. Pollitt, and T. Morris, "Damped blade having a single coating of vibration-damping material," US, US6059533, 2000.

[9] H.-Y. Yen and M.-H. Herman Shen, "Passive vibration suppression of beams and blades using magnetomechanical coating," Journal of Sound and Vibration, vol. 245, no. 4, pp. 701-714, 2001.

[10] G. Gregori, L. Lì, J. A. Nychka, and D. R. Clarke, "Vibration damping of superalloys and thermal barrier coatings at hightemperatures," Materials Science and Engineering A, vol. 466, no. 1-2, pp. 256-264, 2007.

[11] F. Ivancic and A. Palazotto, "Experimental considerations for determining the damping coefficients of hard coatings," Journal of Aerospace Engineering, vol. 18, no. 1, pp. 8-17, 2005.

[12] C. Blackwell, A. Palazotto, T. J. George, and C. J. Cross, "The evaluation of the damping characteristics of a hard coating on titanium," Shock and Vibration, vol. 14, no. 1, pp. 37-51, 2007.

[13] J. Green and S. Patsias, "A Preliminary approach for the modeling of a hard damping coating using friction elements," in Proceedings of the 7th National Turbine Engine High Cycle Fatigue Conference, pp. 1-9, 2002.

[14] J. P. Jang, Q. X. Liu, and W. G. Mao, "Finite element analysis of stress field of thermal barrier coating system of cylindrical shell structure under thermal cycling," CCTAM, 2009.
[15] X. Y. Song, H. J. Ren, X. P. Wang, X. J. Li, and Q. K. Han, “Highorder vibration characteristics of rotating thin shells and hardcoating damping effects," Journal of Physics: Conference Series, vol. 448, no. 1, Article ID 012002, 2013.

[16] A. M. Najafov, A. H. Sofiyev, and N. Kuruoglu, "On the solution of nonlinear vibration of truncated conical shells covered by functionally graded coatings," Acta Mechanica, vol. 225, no. 2, pp. 563-580, 2014.

[17] A. H. Sofiyev, "The vibration and buckling of sandwich cylindrical shells covered by different coatings subjected to the hydrostatic pressure," Composite Structures, vol. 117, no. 1, pp. 124-134, 2014.

[18] Z.-M. Li, X.-D. Chen, and H.-D. Yu, "Large-Amplitude vibration analysis of shear deformable laminated composite cylindrical shells with initial imperfections in thermal environments," Journal of Engineering Mechanics, vol. 140, no. 3, pp. 552-565, 2014.

[19] V. K. Singh and S. K. Panda, "Nonlinear free vibration analysis of single/doubly curved composite shallow shell panels," ThinWalled Structures, vol. 85, pp. 341-349, 2014.

[20] P. Ribeiro, "Non-linear modes of vibration of thin cylindrical shells in composite laminates with curvilinear fibres," Composite Structures, vol. 122, pp. 184-197, 2015.

[21] T. R. Mahapatra, V. R. Kar, and S. K. Panda, "Nonlinear free vibration analysis of laminated composite doubly curved shell panel in hygrothermal environment," Journal of Sandwich Structures and Materials, vol. 17, no. 5, pp. 511-545, 2015.

[22] V. R. Kar and S. K. Panda, "Geometrical nonlinear free vibration analysis of FGM spherical panel under nonlinear thermal loading with TD and TID properties," Journal of Thermal Stresses, vol. 39, no. 8, pp. 942-959, 2016.

[23] T. R. Mahapatra and S. K. Panda, "Nonlinear free vibration analysis of laminated composite spherical shell panel under elevated hygrothermal environment: a micromechanical approach," Aerospace Science and Technology, vol. 49, pp. 276-288, 2016.

[24] F. Pellicano, "Vibrations of circular cylindrical shells: theory and experiments," Journal of Sound and Vibration, vol. 303, no. 1-2, pp. 154-170, 2007.

[25] H. Li, W. Sun, Y.-F. Zhang, and Q.-K. Han, "Accurate test of natural frequency of constrained thin cylindrical shell," Journal of Northeastern University, vol. 34, no. 9, pp. 1314-1318, 2013.

[26] P. J. Torvik, "On estimating system damping from frequency response bandwidths," Journal of Sound and Vibration, vol. 330, no. 25, pp. 6088-6097, 2011.

[27] K. F. Chen and Q. Y. Jiao, "Influence of linear interpolation approximation to half power points on the damping estimation precision," Journal of Mechanical Strength, vol. 24, pp. 510-514, 2002.

[28] H. Li, P.-C. Xue, Z. Xu, P.-F. Shen, and B.-C. Wen, "Identification of modal damping of composite cylindrical shell based on sliding-envelop method," Journal of Aerospace Power, vol. 30, no. 12, pp. 2833-2839, 2015.

[29] H. Li, W. Sun, Z. Xu, and Q.-K. Han, "Experimental method of laser rotating sanning to measure mode shapes of constrained thin cylindrical shell," Journal of Vibration and Shock, vol. 33, no. 16, pp. 155-159, 2014.

[30] S. A. Reed, A. N. Palazotto, and W. P. Baker, "An experimental technique for the evaluation of strain dependent material 
properties of hard coatings," Shock and Vibration, vol. 15, no. 6, pp. 697-712, 2008.

[31] F. Ivancic, The Effect of a Hard Coating on the Damping and Fatigue Life of Titanium, Air Force Institute of Technology, Ohio, USA. 


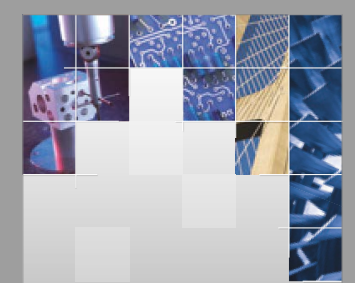

\section{Enfincering}
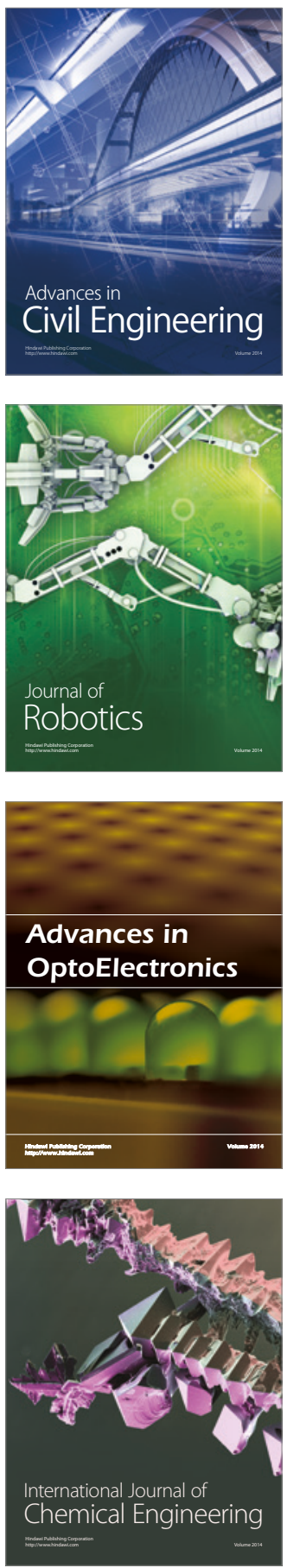

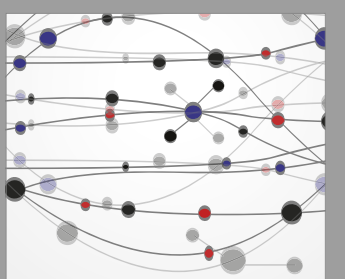

The Scientific World Journal

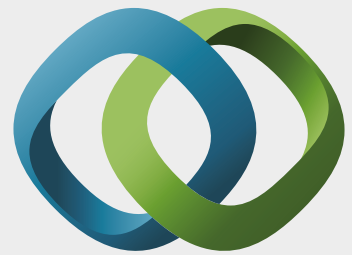

\section{Hindawi}

Submit your manuscripts at

https://www.hindawi.com
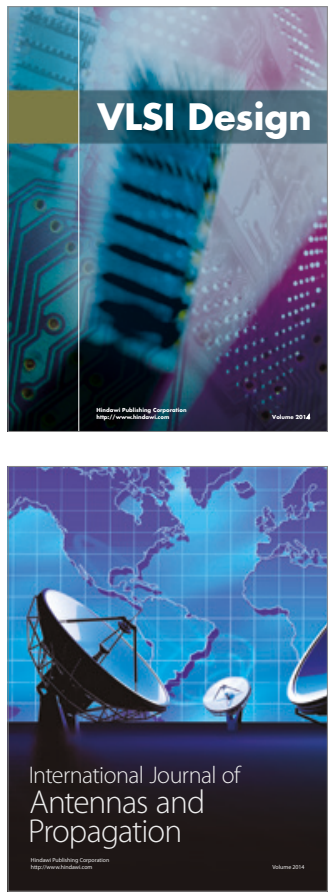

\section{Rotating}

Machinery
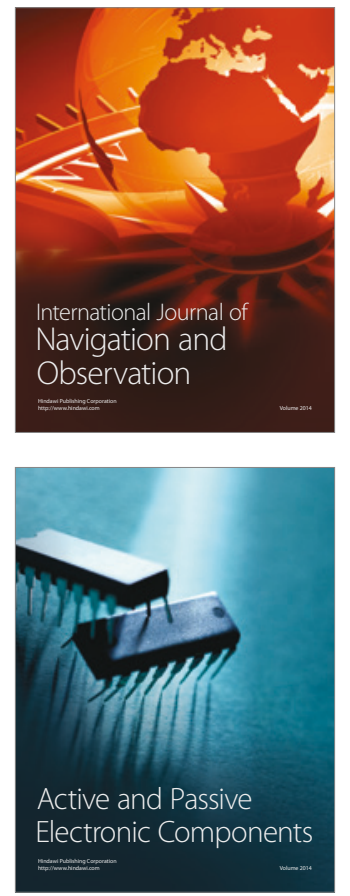
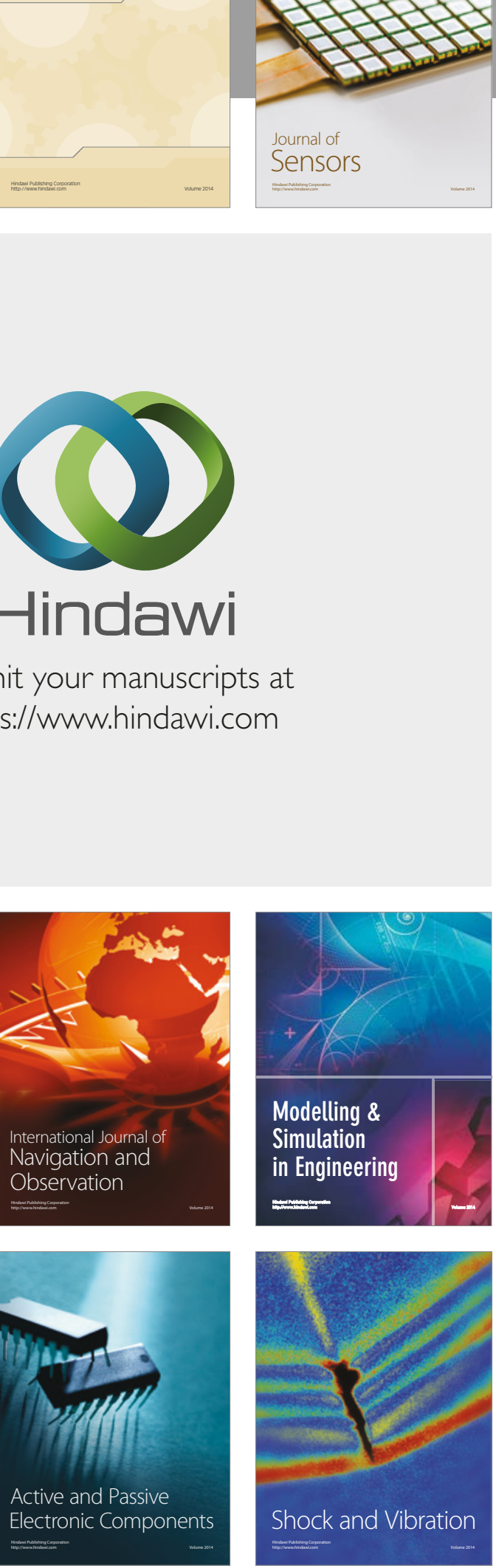
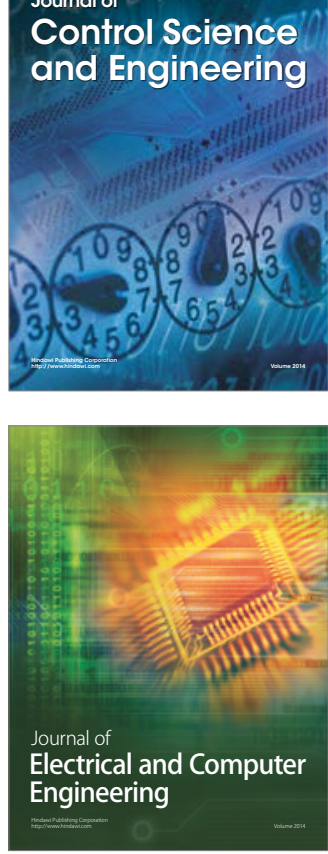

Distributed

Journal of

Control Science

and Engineering
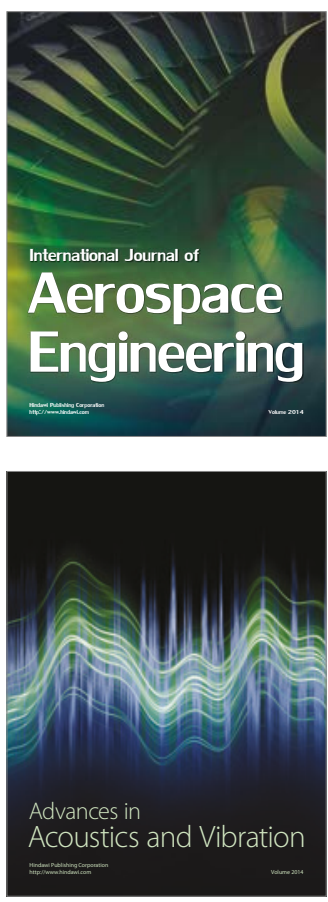

Sensor Networks 